\title{
Multimode Equivalent Network for Boxed Multilayer Arbitrary Planar Circuits
}

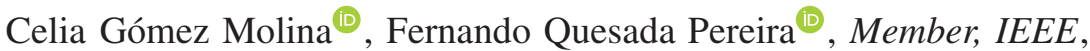 \\ Alejandro Álvarez Melcón ${ }^{\circledR}$, Senior Member, IEEE, Stephan Marini ${ }^{\circledR}$, Senior Member, IEEE, \\ Miguel Ángel Sánchez-Soriano ${ }^{\circledR}$, Senior Member, IEEE, Vicente E. Boria ${ }^{\circledR}$, Fellow, IEEE, \\ and Marco Guglielmi ${ }^{\circledR}$, Life Fellow, IEEE
}

\begin{abstract}
The multimode equivalent network (MEN) formulation has been originally developed for the efficient and accurate analysis of waveguide devices. In this article, we extend the use of the MEN to the analysis of zero-thickness, planar printed circuits in a metallic enclosure. The formulation is developed for metallic areas of arbitrary shape and includes both internal and external ports in the transverse plane to model connections to external components, and coaxial input/output ports. The boundary integral resonant mode expansion (BI-RME) method is used for the analysis of the arbitrary shape metallizations. On this basis, shielded multilayered microstrip circuits of complex geometry are analyzed in the common frame of the MEN technique. To validate the theoretical formulation, several boxed microstrip structures are analyzed, including multilayered configurations with several metallization interfaces, showing good agreement with respect to both other commercial tools, and measurements.
\end{abstract}

Index Terms - Integral equations (IEs), method of moments (MoM), microwave filters, monolithic microwave integrated circuits (MMICs), multimode equivalent networks (MENs), numerical methods, planar junctions.

\section{INTRODUCTION}

$\mathbf{C}$ URRENTLY, computational electromagnetics (CEM) techniques [1], [2] are widely used to save development time and manufacturing costs in the microwave industry. In this context, therefore, new more efficient numerical methods for the design of microwave components are of

Manuscript received December 27, 2019; revised February 17, 2020; accepted March 11, 2020. This work was supported in part by the Spanish Government through the Ministerio de Educación, Cultura y Deporte under Grant FPU15/02883 and in part by the Ministerio de Economía y Competitividad through the sub-projects 4,2 , and 1 of the coordinated project under Grant TEC2016-75934-C4-R. (Corresponding author: Celia Gómez Molina.)

Celia Gómez Molina, Fernando Quesada Pereira, and Alejandro Álvarez Melcón are with the Department of Information Technologies and Communications, Universidad Politécnica de Cartagena, 30202 Cartagena, Spain (e-mail: celia.gomez@upct.es; fernando.quesada@upct.es; alejandro. alvarez@upct.es).

Stephan Marini and Miguel Ángel Sánchez-Soriano are with the Department of Physics, Systems Engineering and Signal Theory, Universidad de Alicante, 03690 Alicante, Spain (e-mail: smarini@ua.es).

Vicente E. Boria and Marco Guglielmi are with the Institute of Telecommunication and Multimedia Applications, Universidad Politécnica de Valencia, 46022 Valencia, Spain (e-mail: vboria@dcom.upv.es; marco.guglielmi@iteam.upv.es).

Color versions of one or more of the figures in this article are available online at http://ieeexplore.iee.org.

Digital Object Identifier 10.1109/TMTT.2020.2984230 great interest in the electromagnetics research area. A large variety of numerical techniques have indeed been reported in the technical literature (see [3]-[5]), and are also implemented in commercial software tools such as ANSYS high-frequency structure simulator (HFSS), CST Studio Suite, FEST3D, or Advanced Design System (ADS). Some of these techniques [3], [4] can perform the analysis of arbitrary microwave structures, at the expense, most of the time, of consuming high computational resources. Other techniques, on the other hand, are based on modal methods and integral equations (IEs) [6], and can analyze a more limited variety of geometries, but with important reduction in computational time.

The analysis of planar structures, using an electric field IE (EFIE), has already been discussed in the past by several authors [7]-[9]. Melcón et al. [7] analyzed boxed microwave circuits composed of rectangular printed metallizations. In [8], the boundary integral resonant mode expansion (BI-RME) method was efficiently used for the analysis of planar structures with complex geometries. In [9], on the other hand, arbitrary geometries are treated using subsectional rooftop basis functions, instead of entire domain basis functions as in [7] and [8]. Finally, the analysis of open microstrip structures based on EFIE formulations using closed-form Green's functions, where rooftop or piecewise-sinusoidal (PS) basis functions are used, is treated in great detail in other related works [10]-[14].

In this context, therefore, the objective of this article is to extend the state-of-the-art of the analysis of planar structures by discussing an alternative IE technique based on the multimode equivalent network (MEN) formulation [15]. This technique begins with the individual MEN characterization of the different planar circuits (or discontinuities) that compose the structure under study. The various MENs are then properly combined to perform the analysis of the complete device. For each discontinuity, the formulation starts by imposing the corresponding boundary conditions for the electromagnetic fields to obtain the relevant IEs. A circuit interpretation of the solutions of the IEs leads to an equivalent network, where all modal interactions are rigorously accounted for through a generalized impedance coupling matrix.

The MEN technique was initially developed for the analysis of waveguide components [16]. Its usefulness and efficiency are shown, for instance, in [17]-[19]. The first contribution 
in the context of the MEN formulation for the analysis of planar structures can be found in [20], where the authors derived the electric and magnetic MEN formulations applied to the analysis of zero-thickness metal-strip gratings seen as obstacles or apertures, respectively. In these original formulations, the structures are excited by guided modes on both sides of the discontinuity under study. This allows the analysis of useful zero-thickness waveguide discontinuities, and frequency selective surfaces (FSSs) (as treated in [21]). However, these original formulations do not include the ports in the transverse plane that are needed for the excitation of printed microstrip and multilayer circuits. The first works to do so with the MEN technique, to the authors' knowledge, are [22] and [23]. In these works, the authors derived the MEN formulation for the analysis of multilayer planar circuits composed of rectangular printed metallizations using an electric field formulation. In addition, the formulation in [22] and [23] is still limited to areas with a rectangular shape, where entire domain basis functions are known analytically.

In this article, we present an extension of the method proposed in [22] and [23] to the case of multilayer circuits that are composed of printed metallic patches with arbitrary shape (like the one shown in Fig. 1). For this purpose, the BI-RME method [24], [25] is used to obtain the basis functions needed to solve the IEs with complex (arbitrarily shaped) planar geometries. Note that the BI-RME method has been previously used in MEN formulations applied to the analysis of waveguide discontinuities [25]. However, this is the first time that a MEN formulation is combined with BI-RME for the analysis of arbitrarily shaped printed metallizations in a multilayered environment. Another important difference with respect to [22] and [23] is that we use a magnetic field formulation to derive the fundamental IEs. Following this approach, the unknowns of the problem are magnetic currents defined in the aperture, instead of the electric currents induced on the metallic areas. This formulation, therefore, complements the one presented in [22], and allows the analysis of complex multilayered printed circuits by combining electric and magnetic field formulations. The idea of combining electric and magnetic formulations was also proposed in [20], in the context of the analysis of zero-thickness discontinuities inside waveguides. With the extension proposed in this article, the same concept can now be applied to the analysis of microstrip and multilayered printed circuits. It is also important to note that the ports modeled in this article allows to use the MEN technique with coaxial input/output ports, and to connect external lumped-element components to the printed circuits being analyzed.

The main difference between the formulation that we discuss in this article, and the EFIE techniques discussed in [7]-[9], is that we solve the problem in the frame of the MEN representation, so that the analysis of planar circuits can be seamlessly integrated with the analysis of complex waveguide structures and antennas. A further advantage of the formulation that we propose is related to the analysis of multilayer circuits containing several layers of metallization. In the direct EFIE implementations discussed in [7]-[9], all the unknowns of the problem are included in one global IE. This can lead to matrices of very large sizes when several
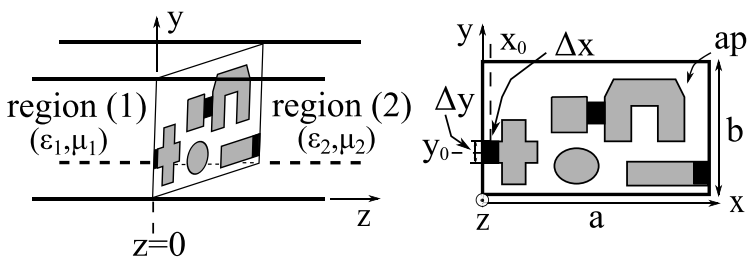

Fig. 1. 2-D zero-thickness printed circuit in planar technology. The gray areas correspond to the printed metallizations and the dark areas to the excitation and internal ports, both placed on the transverse $(z=0)$ plane. The media on both sides of the discontinuity can be different, for instance the substrate and the air of a microstrip structure. The size of the ports along the $x$-and $y$-axes is denoted as $\Delta x$ and $\Delta y$, respectively.

layers are analyzed. The MEN technique that we discuss, on the other hand, formulates one separate IE to characterize each discontinuity with a different multimode coupling matrix. All discontinuities are then coupled together by cascading the different multimode impedance coupling matrices. The result is that, instead of one large global problem, we solve a number of smaller problems that are then properly combined at network level.

We should emphasize that the structures addressed in this article are classic multilayered shielded planar circuits. However, the work in [22], together with this article, can be considered as the first applications of the MEN technique to the analysis of this kind of structures. The extension of the MEN technique to more complex structures including, for instance, anisotropic sheets, will be considered in the future research.

This article is organized as follows. In Section II, we describe the theoretical details of the formulation. In particular, we put special emphasis on how to integrate ports in the transverse plane with a magnetic field formulation. To validate the theory, numerical results for several printed microstrip structures (including internal ports and also more than one metallic layer) are then discussed in Section III. Finally, the main conclusions are outlined in Section IV.

\section{Formulation}

In this section, we formulate the rigorous MEN representation of a 2-D zero-thickness obstacle consisting of arbitrarily shaped printed metallizations (see Fig. 1). The theoretical derivations also include internal and external lumped ports in the transverse plane, as shown in Fig. 1 (dark areas). An important difference with respect to [22] is that in this article, we use the magnetic field boundary condition to derive the basic MEN. This allows for the rigorous inclusion in the MEN of any number of ports in the transverse plane.

For the sake of simplicity, however, just one lumped excitation port is included explicitly in our formulation. The extension to more than one port (either external, or internal for interconnection purposes) is straightforward. It is important to note that, from now, we use text in bold to indicate vectors and text in bold with bars to indicate matrices.

The formulation starts by imposing the continuity of the tangential components of the magnetic field $\mathbf{H}_{t}^{(\delta)}$ in the aperture at $z=0$, including the excitation contribution

$$
\mathbf{H}_{t}^{(1)}(s)-\mathbf{H}_{t}^{(2)}(s)=\mathbf{z}_{0} \times \mathbf{J}_{\mathrm{exc}}(s)
$$


where $\delta=1$ or $\delta=2$ for $z<0$ or $z>0$, respectively, and $s=(x, y)$ indicates a point in the junction cross section of Fig. 1. In (1), the excitation port has been accounted for by including the surface electric current density $\mathbf{J}_{\text {exc }}$. We stress that in this article, we use a pulse excitation model [9]. It has, in fact, been shown that this model can correctly represent the coaxial connectors normally used as excitation in real shielded planar structures. A delta-gap model [26] has also been used for this purpose. However, the difference between pulse and a delta-gap model is that the pulse model includes also the effect of the width $(\Delta y)$ along the $y$-axis in the excitation. Using the pulse model, we assume to apply at the excitation port a current $I_{0}$ that is constant along the width of a pulse function. Therefore, the resulting expression for the surface electric current density $\mathbf{J}_{\text {exc }}$ is

$$
\mathbf{J}_{\mathrm{exc}}(s)=-I_{0} \frac{1}{\Delta y} \prod\left(\frac{x-x_{0}}{\Delta x}\right)\left(\frac{y-y_{0}}{\Delta y}\right) \mathbf{x}_{0}
$$

where $\left(x_{0}, y_{0}\right)$ are the coordinates of the center of the pulse and $\Delta x$ and $\Delta y$ are the widths of the pulse in the $x$-axis and $y$ axis, respectively (see Fig. 1). Note that since in this example the input line is directed along the $x$-axis, the current exciting the circuit only has $x$-component. However, the scattered field in the aperture is modeled with all vector components. Therefore, the induced currents in the metallizations will be correctly modeled with all needed vector components.

The amplitude of the pulse is normalized with the factor $1 / \Delta y$ to ensure that the power flow $P_{\text {exc }}$ defined as

$$
P_{\mathrm{exc}}=\frac{1}{2} \int_{S_{\mathrm{exc}}}\left(\mathbf{E}_{\mathrm{exc}} \times \mathbf{H}_{\mathrm{exc}}^{*}\right) \cdot \mathbf{z}_{0} d S
$$

is equal to that associated with the equivalent transmission line $P_{0}$ defined as

$$
P_{0}=\frac{1}{2} V_{0} I_{0}^{*}
$$

where $V_{0}$ is the voltage across the port, and $I_{0}$ is the port current. Moreover, the integral in (3) extends to the pulse area denoted as $\left(S_{\mathrm{exc}}=\Delta x \Delta y\right)$.

By using this excitation model, and the well-known modal expansion formalism [15], (1) can be written as

$$
\begin{aligned}
\sum_{m=1}^{+\infty} I_{m}^{(1)} \mathbf{h}_{m}^{(1)}(s)-\sum_{m=1}^{+\infty} I_{m}^{(2)} \mathbf{h}_{m}^{(2)}(s) & \\
& =\frac{-I_{0}}{\Delta y} \prod\left(\frac{x-x_{0}}{\Delta x}\right)\left(\frac{y-y_{0}}{\Delta y}\right) \mathbf{y}_{0}
\end{aligned}
$$

where $\mathbf{h}_{m}^{(\delta)}(s)$ represents the magnetic vector mode function of mode $m$ in the medium $(\delta)$, and $I_{m}^{(\delta)}$ refers to the modal currents. It is important to note that $m$ refers to all TE and TM modes in the rectangular waveguide of cross section $(a \times b)$ of Fig. 1.

To continue, we now separate the modes which contribute to the storage of energy (localized modes) from the modes that contribute to the energy exchange between cascaded discontinuities (accessible modes) [27]. This is done by splitting the infinite modal series into two terms, thus obtaining

$$
\begin{aligned}
& \sum_{n=1}^{N(1)} I_{n}^{(1)} \mathbf{h}_{n}^{(1)}(s)-\sum_{n=1}^{N(2)} I_{n}^{(2)} \mathbf{h}_{n}^{(2)}(s) \\
& \quad+\frac{I_{0}}{\Delta y} \prod\left(\frac{x-x_{0}}{\Delta x}\right)\left(\frac{y-y_{0}}{\Delta y}\right) \mathbf{y}_{0} \\
& =\sum_{m=N(1)+1}^{+\infty} \frac{V_{m}^{(1)}}{Z_{m}^{(1)}} \mathbf{h}_{m}^{(1)}(s)+\sum_{m=N(2)+1}^{+\infty} \frac{V_{m}^{(2)}}{Z_{m}^{(2)}} \mathbf{h}_{m}^{(2)}(s)
\end{aligned}
$$

where $N(\delta)$ is the number of accessible modes in each region.

In (6), $V_{m}^{(\delta)}$ represents the modal voltage and $Z_{m}^{(\delta)}$ the characteristic modal impedance in medium $(\delta)$. Note that, for the sake of clarity, the subscript on the left-hand side in (6) has been changed from $m$ to $n$.

We now recognize that, since the metallic circuit is zerothickness, and the waveguide cross section in region (1) is identical to the one in region (2), the magnetic vector mode functions in region (1) $\left(\mathbf{h}_{m}^{(1)}\right)$ are identical to the ones in region (2) $\left(\mathbf{h}_{m}^{(2)}\right)$. Then, knowing that $\mathbf{h}_{m}^{(1)}(s)=\mathbf{h}_{m}^{(2)}(s)=\mathbf{h}_{m}(s)$ and $V_{m}^{(1)}=V_{m}^{(2)}=V_{m},(6)$ is transformed into

$$
\begin{aligned}
\sum_{n=1}^{N} \bar{I}_{n} \mathbf{h}_{n}(s)+I_{0} \frac{1}{\Delta y} \prod\left(\frac{x-x_{0}}{\Delta x}\right) & \left(\frac{y-y_{0}}{\Delta y}\right) \mathbf{y}_{0} \\
= & \sum_{m=N+1}^{+\infty} V_{m} Y_{m}^{T} \mathbf{h}_{m}(s)
\end{aligned}
$$

where $\bar{I}_{n}$ represents the total modal current at the interface, defined as $\bar{I}_{n}=I_{n}^{(1)}-I_{n}^{(2)}$. Also, $Y_{m}^{T}$ is the total characteristic modal admittance defined as $Y_{m}^{T}=Y_{m}^{(1)}+Y_{m}^{(2)}$, where $Y_{m}^{(\delta)}$ represents the characteristic modal admittance of mode $m$ in the medium $(\delta)$. Note that the number of accessible modes in both regions are assumed to be equal, $N(1)=N(2)=N$, however, a different number of accessible modes can also be easily considered.

To continue the formulation, we now recall the following relation between the modal voltage $V_{m}$ and the electric field in the aperture $\mathbf{E}$ :

$$
V_{m}=\int_{\mathrm{ap}}\left[\mathbf{z}_{0} \times \mathbf{E}\left(s^{\prime}\right)\right] \cdot \mathbf{h}_{m}^{*}\left(s^{\prime}\right) d s^{\prime}
$$

where the integral extends over the entire aperture (ap) at the junction (see Fig. 1) and $d s^{\prime}=d x^{\prime} d y^{\prime}$.

Due to the linearity of the problem, the unknown transverse electric field in the aperture $\left[\mathbf{z}_{0} \times \mathbf{E}\left(s^{\prime}\right)\right]$ must be linearly proportional to the excitation, so that it can be expanded into a sum of partial surface magnetic current densities $\mathbf{M}_{n}\left(s^{\prime}\right)$ and $\mathbf{M}_{0}\left(s^{\prime}\right)$ as follows:

$$
\left[\mathbf{z}_{0} \times \mathbf{E}\left(s^{\prime}\right)\right]=\sum_{n=1}^{N} \bar{I}_{n} \mathbf{M}_{n}\left(s^{\prime}\right)+I_{0} \mathbf{M}_{0}\left(s^{\prime}\right)
$$

where $\mathbf{M}_{n}$ and $\mathbf{M}_{0}$ are the unknown functions of our problem. Substituting now (9) into (8), and by using the resulting equation in (7), we can finally obtain the fundamental IEs 
of our problem

$$
\begin{aligned}
\mathbf{h}_{n}(s) & =\int_{\mathrm{ap}} \mathbf{M}_{n}\left(s^{\prime}\right) \cdot \overline{\mathbf{K}}\left(s, s^{\prime}\right) d s^{\prime} \\
\frac{1}{\Delta y} \prod\left(\frac{x-x_{0}}{\Delta x}\right)\left(\frac{y-y_{0}}{\Delta y}\right) \mathbf{y}_{0} & =\int_{\mathrm{ap}} \mathbf{M}_{0}\left(s^{\prime}\right) \cdot \overline{\mathbf{K}}\left(s, s^{\prime}\right) d s^{\prime}
\end{aligned}
$$

where $\overline{\mathbf{K}}\left(s, s^{\prime}\right)$ is the kernel of the IEs

$$
\overline{\mathbf{K}}\left(s, s^{\prime}\right)=\sum_{m=N+1}^{+\infty} Y_{m}^{T} \mathbf{h}_{m}^{*}\left(s^{\prime}\right) \mathbf{h}_{m}(s) .
$$

In order to increase the efficiency of this formulation, we use the Kummer's transformation, as already described in [28], for the acceleration of the kernel of the IEs. Using this strategy, the kernel is divided into static and dynamic parts. As a result, the dynamic part, that needs to be recomputed per each frequency point, exhibits now a faster convergence rate.

To complete the rigorous network formulation, (9) is used in (8) to write the modal voltages in terms of the modal currents

$$
\begin{gathered}
V_{0}=Z_{0,0} I_{0}+\sum_{n=1}^{N} Z_{0, n} \bar{I}_{n} \\
V_{m}=Z_{m, 0} I_{0}+\sum_{n=1}^{N} Z_{m, n} \bar{I}_{n}
\end{gathered}
$$

where $Z_{0,0}, Z_{0, n}, Z_{m, 0}$, and $Z_{m, n}$ are the elements of the generalized impedance coupling matrix $\overline{\mathbf{Z}}$ that are given by

$$
\begin{aligned}
Z_{0,0} & =\int_{\mathrm{ap}} \mathbf{M}_{0}\left(s^{\prime}\right) \cdot\left(\frac{1}{\Delta y} \prod\left(\frac{x-x_{0}}{\Delta x}\right)\left(\frac{y-y_{0}}{\Delta y}\right) \mathbf{y}_{0}\right) d s^{\prime} \\
Z_{0, n} & =\int_{\mathrm{ap}} \mathbf{M}_{n}\left(s^{\prime}\right) \cdot\left(\frac{1}{\Delta y} \prod\left(\frac{x-x_{0}}{\Delta x}\right)\left(\frac{y-y_{0}}{\Delta y}\right) \mathbf{y}_{0}\right) d s^{\prime} \\
Z_{m, 0} & =\int_{\mathrm{ap}} \mathbf{M}_{0}\left(s^{\prime}\right) \cdot \mathbf{h}_{m}^{*}\left(s^{\prime}\right) d s^{\prime} \\
Z_{m, n} & =\int_{\mathrm{ap}} \mathbf{M}_{n}\left(s^{\prime}\right) \cdot \mathbf{h}_{m}^{*}\left(s^{\prime}\right) d s^{\prime} .
\end{aligned}
$$

The resulting multimode impedance coupling matrix is of size $(N+1) \times(N+1)$. We can therefore write

$$
\overline{\mathbf{Z}}=\left[\begin{array}{ccccc}
Z_{0,0} & Z_{0,1} & Z_{0,2} & \cdot & Z_{0, N} \\
Z_{1,0} & Z_{1,1} & Z_{1,2} & \cdot & Z_{1, N} \\
\cdot & \cdot & & & \cdot \\
\cdot & & \cdot & & \cdot \\
\cdot & & & \cdot & \cdot \\
Z_{N, 0} & Z_{N, 1} & Z_{N, 2} & \cdot & Z_{N, N}
\end{array}\right] .
$$

In network terms, we finally obtain the representation shown in Fig. 2.

In order to solve the IEs in (10) and (11), we now expand the unknown partial magnetic current functions $\mathbf{M}_{n}\left(s^{\prime}\right)$ and $\mathbf{M}_{0}\left(s^{\prime}\right)$ using the method of moments (MoM) [15]. In particular, we use the magnetic vector mode functions in the aperture at the plane of the junction both as basis $\left(\mathbf{h}_{k}^{B}\right)$ and test $\left(\mathbf{h}_{i}^{B}\right)$ functions, applying the Galerkin method [15]. Following

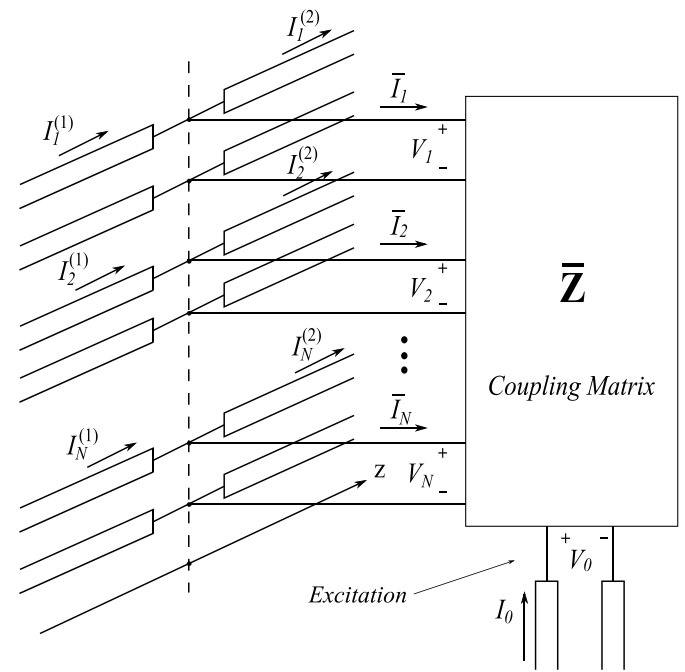

Fig. 2. MEN (generalized $Z$-matrix) representation for the zero-thickness discontinuity with arbitrarily shaped metallic areas, shown in Fig. 1, including one port in the transverse plane.

this approach, the unknowns $\mathbf{M}_{n}\left(s^{\prime}\right)$ and $\mathbf{M}_{0}\left(s^{\prime}\right)$ can finally be written as:

$$
\mathbf{M}_{0}\left(s^{\prime}\right)=\sum_{k=1}^{N_{b}} \alpha_{0, k} \mathbf{h}_{k}^{B}\left(s^{\prime}\right) ; \quad \mathbf{M}_{n}\left(s^{\prime}\right)=\sum_{k=1}^{N_{b}} \alpha_{n, k} \mathbf{h}_{k}^{B}\left(s^{\prime}\right)
$$

where $N_{b}$ is the numerical parameter that determines the number of basis and test functions used in the MoM procedure, and $\alpha_{0, k}$ and $\alpha_{n, k}$ are the unknown coefficients of the expansions.

Applying the test procedure, the IEs in (10) and (11) are finally transformed into the following system of linear equations:

$$
\begin{aligned}
C_{i, n} & =\sum_{k=1}^{N_{b}} \alpha_{n, k} \sum_{m=N+1}^{+\infty} Y_{m}^{T} C_{k, m} C_{i, m} \\
C_{i, 0} & =\sum_{k=1}^{N_{b}} \alpha_{0, k} \sum_{m=N+1}^{+\infty} Y_{m}^{T} C_{k, m} C_{i, m} .
\end{aligned}
$$

The coefficients $C_{k, m}$ and $C_{i, m}$ represent the coupling integrals between the localized modes of the rectangular waveguide, and the aperture modes used as basis and test functions, respectively

$$
\begin{aligned}
C_{k, m} & =\int_{\mathrm{ap}} \mathbf{h}_{k}^{B}\left(s^{\prime}\right) \cdot \mathbf{h}_{m}^{*}\left(s^{\prime}\right) d s^{\prime} \\
C_{i, m} & =\int_{\mathrm{ap}} \mathbf{h}_{i}^{B}(s) \cdot \mathbf{h}_{m}(s) d s .
\end{aligned}
$$

The terms $C_{i, n}$ are the coupling integrals between the accessible modes of the rectangular waveguide and the aperture modes used as test functions

$$
C_{i, n}=\int_{\mathrm{ap}} \mathbf{h}_{i}^{B}(s) \cdot \mathbf{h}_{n}(s) d s .
$$

The term $C_{i, 0}$, on the other hand, refers to the coupling integral between the test functions and the excitation pulse

$$
C_{i, 0}=\int_{\mathrm{ap}} \mathbf{h}_{i}^{B}(s) \cdot\left(\frac{1}{\Delta y} \prod\left(\frac{x-x_{0}}{\Delta x}\right)\left(\frac{y-y_{0}}{\Delta y}\right) \mathbf{y}_{0}\right) d s .
$$


It is important to mention that, since the metallization at the junction is arbitrarily shaped, the coupling integrals in (22), (23), and (24), that are related to the modes in the aperture, can conveniently be computed numerically using the BI-RME method [24], [25]. The coupling integrals $C_{i, 0}$ can then be computed analytically using the numerical expansion in terms of rectangular waveguide modes of the basis functions $\left[\mathbf{h}_{i}^{B}(s)\right]$, which are also provided by the BI-RME method. To illustrate the procedure, let $\mathbf{h}_{m}(s)$ be the rectangular waveguide modes used to expand the arbitrary aperture $\operatorname{modes} \mathbf{h}_{i}^{B}(s)$. Using the BI-RME method we obtain the following expression:

$$
\mathbf{h}_{i}^{B}(s)=\sum_{m=1}^{N_{k}} C_{i, m} \mathbf{h}_{m}(s)
$$

where $C_{i, m}$ are the expansion coefficients calculated by BIRME [the same ones as those in (23)]. According to (26), the coupling integral in (25) can be computed as

$$
\begin{aligned}
C_{i, 0}=\sum_{m=1}^{N_{k}} C_{i, m} \cdot \int_{\mathrm{ap}} \mathbf{h}_{m}(s) \\
\quad \cdot\left(\frac{1}{\Delta y} \prod\left(\frac{x-x_{0}}{\Delta x}\right)\left(\frac{y-y_{0}}{\Delta y}\right) \mathbf{y}_{0}\right) d s
\end{aligned}
$$

where the remaining integral is now analytical.

According to this notation, the elements of the generalized impedance coupling matrix $\overline{\mathbf{Z}}$ can be rewritten in a more compact form as

$$
\begin{aligned}
& Z_{0,0}=\sum_{k=1}^{N_{b}} \alpha_{0, k} C_{k, 0} ; \quad Z_{0, n}=\sum_{k=1}^{N_{b}} \alpha_{n, k} C_{k, 0} \\
& Z_{m, 0}=\sum_{k=1}^{N_{b}} \alpha_{0, k} C_{k, m} ; \quad Z_{m, n}=\sum_{k=1}^{N_{b}} \alpha_{n, k} C_{k, m} .
\end{aligned}
$$

Note that, with this formulation, we have imposed the boundary conditions that the tangential magnetic field must satisfy at the aperture using (1). The continuity of the tangential components of the electric field is imposed implicitly when the modal voltages on both sides of the discontinuity are assumed to be equal $V_{m}^{(1)}=V_{m}^{(2)}=V_{m}$ (see Fig. 2).

It is also important to note that the circuit in Fig. 1 is only an example of a possible microwave circuit. The formulation presented in this article is not restricted to any particular shape. In fact, the shape can be arbitrarily thanks to the use of the BI-RME method [24], [25]. In addition, we should emphasize that the formulation we discuss is valid also for isotropic substrates. The analysis of anisotropic substrates would, however, require to change the kernel of the IEs. Furthermore, the formulation is strictly valid only for zerothickness metallization (or in practice, for a thickness that is much smaller than the wavelength). For thicker structures, the formulation presented is not valid and an alternative formulation based on thick discontinuities must be developed.

\section{A. Application to Lossy Microstrip Circuits}

In this section, the MEN formulation is particularized to the analysis of typical shielded printed microstrip circuits. In this

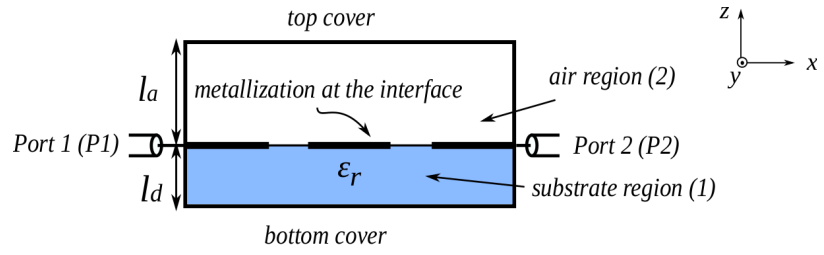

Fig. 3. Schematic of the shielded microstrip printed circuit under study, excited with two coaxial connectors.

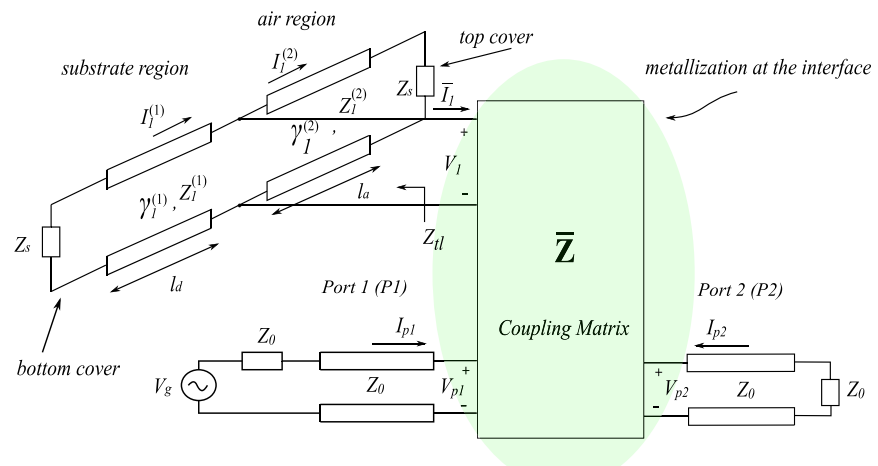

Fig. 4. Equivalent network representation for the microstrip circuit shown in Fig. 3 using the MEN formalism. The metallization at the interface is characterized by its equivalent impedance coupling matrix. Just one accessible mode is considered, which is characterized with transmission lines in the air $\left(l_{a}\right)$ and dielectric $\left(l_{d}\right)$ regions, terminated with the impedance $Z_{s}$ representing losses in the top and bottom covers of the cavity box (see Fig. 3). The coaxial excitations are represented by transmission lines of characteristic impedance $Z_{0}$.

case, one single metallization between two different media, will be discussed together with two lumped ports, to represent the coaxial input/output connectors. For this structure, region (2) in Fig. 1 is air, while region (1) represents the dielectric substrate. A schematic of the structure under analysis is shown in Fig. 3. The inclusion of losses in the structure is also discussed.

Once we have characterized the metallization at the interface using the MEN technique, the modes in the air region (height $l_{a}$ ) and in the substrate (height $l_{d}$ ) are represented with transmission lines on both sides of the discontinuity (see Fig. 2). As a result, the equivalent circuit of Fig. 3 becomes the one shown in Fig. 4. As we can see in Fig. 4, the transmission lines that represent the accessible modes are terminated by a surface impedance $Z_{s}$ that models the losses in the top/bottom covers of the shielding cavity [29]. Thus, $Z_{s}$ can be zero for a perfect electric conductor (PEC) box, or, if the box is considered to be lossy, it can be computed with

$$
Z_{s}=(1+j) \sqrt{\frac{w \mu}{2 \sigma_{\text {box }}}} .
$$

In this expression $\sigma_{\text {box }}$ is the finite conductivity of the metallic shielding box. Moreover, $w$ is the angular frequency and $\mu$ is the permeability.

In addition, losses in the substrate [region (1)] can be taken into account by using a complex propagation constant in the transmission line model of each mode

$$
\gamma_{m}^{(1)}=\sqrt{k_{c m}^{2}-k_{0}^{2} \epsilon^{\prime}(1-j \tan \delta)}=\alpha_{d_{m}}^{(1)}+j \beta_{m}^{(1)}
$$


where $k_{c m}$ is the cutoff wavenumber of the mode $m$ and $k_{0}$ is the free space wavenumber. In (30), the loss tangent $(\tan \delta)$ models the losses in the dielectric substrate.

Losses in the lateral metallic walls of the box can also be considered by adding an attenuation term $\alpha_{c_{m}}$, due to the finite conductivity of the lateral walls of the box, to the propagation constants of the transmission lines $\gamma_{m}^{(1)}=\alpha_{c_{m}}^{(1)}+\alpha_{d_{m}}^{(1)}+j \beta_{m}^{(1)}$. This attenuation term can be calculated as described in [16] for each mode propagating in the rectangular waveguide.

The last contribution to the losses in the structure of Fig. 3 is due to the printed metallizations. In [30], a formulation was developed to account for these losses in the impedance matrix of the discontinuity. However, in [30] the authors show that the magnetic formulation does not offer accurate results, while increasing the computational time. Furthermore, in most practical microstrip circuits, the most important contributions to the losses come from the dielectric layer(s) and from the metallic enclosure. At the end, for an accurate prediction of the losses, the fine tuning of the various loss constants it is almost always required. In this context, therefore, although theoretically possible, the exact computation of the losses in the metallization is not included in this article. The small contribution to the total losses due to the metallic circuit(s) can be very effectively accounted for by the losses in the dielectric(s), and the box.

An additional very important point that must be noted is that, in this structure, as shown in Fig. 4, we only need to consider one accessible mode. This is because the structure contains only one metallic interface, and therefore the connection to other MENs is not required. This approach is rigorous, if both localized and accessible modes are loaded with the total impedance $Z_{t l}$ shown in Fig. 4. As a result, the mesh equations for the equivalent network of Fig. 4 are written in matrix form as

$$
\left[\begin{array}{c}
V_{p 1} \\
V_{p 2} \\
V_{1}
\end{array}\right]=\left[\begin{array}{ccc}
Z_{p 1, p 1} & Z_{p 1, p 2} & Z_{p 1,1} \\
Z_{p 2, p 1} & Z_{p 2, p 2} & Z_{p 2,1} \\
Z_{1, p 1} & Z_{1, p 2} & Z_{1,1}
\end{array}\right] \cdot\left[\begin{array}{c}
I_{p 1} \\
I_{p 2} \\
\bar{I}_{1}
\end{array}\right]
$$

where only the interactions between the transversal ports $P 1$ and $P 2$ and the first accessible mode are considered. Recall that, for this structure, the input/output coaxial transitions, shown in Fig. 3, are represented by the pulse excitations and the transmission line ports of characteristic impedance $Z_{0}$ shown in the equivalent network of Fig. 4.

To obtain the S-parameters from the equivalent network in Fig. 4, one of the pulse ports is loaded with the port impedance (usually $Z_{0}=50 \Omega$ ), and the other one is taken as the excitation. Since the equivalent impedance coupling matrix $\overline{\mathbf{Z}}$ relates the voltages and the currents in the network ports, as shown in (31), we can solve the system to obtain the input admittance in the excitation port $\left(Y_{\text {in }}=I_{p 1} / V_{p 1}\right)$, and the transadmittance between the input and output ports, $P 1$ and $P 2\left(Y_{\text {tran }}=I_{p 2} / V_{p 1}\right)$. With this information, the S-parameters can be calculated as

$$
\begin{aligned}
S_{11} & =\frac{1-Z_{0} Y_{\text {in }}}{1+Z_{0} Y_{\text {in }}} \\
S_{21} & =Y_{\operatorname{tran}} \frac{\left(-2 Z_{0}\right)}{1+Z_{0} Y_{\text {in }}} .
\end{aligned}
$$

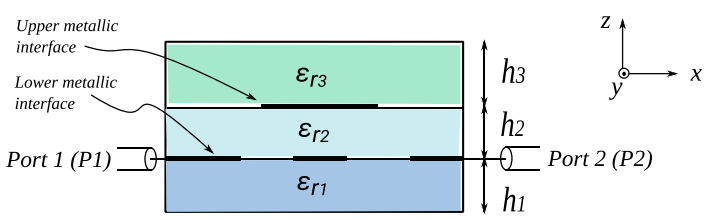

Fig. 5. Circuit containing two metallic interfaces and three dielectric substrates, excited with two coaxial connectors in the lower metallization level.

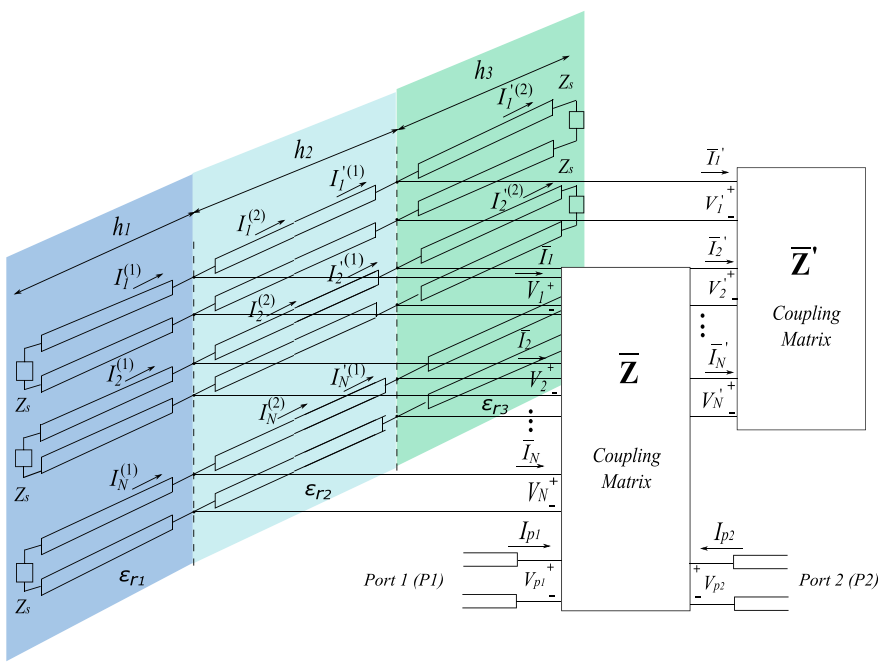

Fig. 6. MEN of the multilayer geometry in Fig. 5.

\section{B. Application to Multilayer Structures}

In this section, we illustrate the use of the MEN formulation described in Section II-A to analyze boxed planar circuits that are composed of two metallic interfaces and three dielectric substrates, as the one shown in Fig. 5. Following the same strategy, the extension to an arbitrary number of dielectric and circuit layers is straightforward.

As in [23], the idea is to analyze separately all the planar discontinuities (metallic interfaces) of the circuit under study, using the formulation that we described. Once the different individual equivalent networks are computed, they can be simply cascaded as shown in Fig. 6. In this network, the transmission lines represent the accessible modes in each dielectric layer. The impedance coupling matrices represent the different printed metallic circuits shown in Fig. 5. As we can see, two coaxial connectors are used as input and output ports. The connectors are modeled by two transverse ports $P 1$ and $P 2$ in the first equivalent network shown in Fig. 6 (lower planar circuit). Again, as we can see, the top and bottom covers of the box are modeled by terminating the transmission lines representing substrates $1\left(h_{1}, \epsilon_{r 1}\right)$ and $3\left(h_{3}, \epsilon_{r 3}\right)$ with a surface impedance $Z_{s}$. As in Section II-A, this impedance models the losses in the top and bottom covers of the box, and is computed using (29).

Note that in our formulation we use TE and TM modes which are transverse with respect to the axis perpendicular to the dielectric layers ( $z$-axis in Fig. 5). These modes do not couple at the interface between two different dielectric layers. Therefore, if no metallic obstacle is present at a dielectric interface, there are no coupling effects between the modes at 


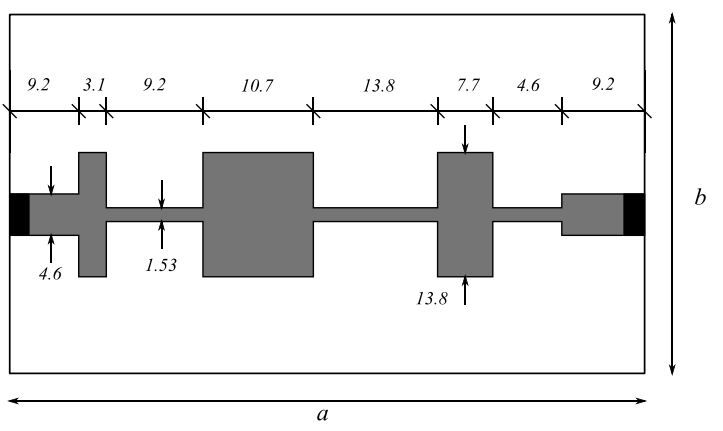

Fig. 7. Lowpass microstrip filter under study. The dimensions are in $\mathrm{mm}$. The dimensions of the shielding box are: $a \times b=67.5 \mathrm{~mm} \times 67.5 \mathrm{~mm}$. With respect to Fig. 3, the value of $l_{a}$ is $9.83 \mathrm{~mm}$. The dielectric relative permittivity is $\varepsilon_{r}=2.33$ and the substrate thickness is $l_{d}=1.57 \mathrm{~mm}$. The filter is centered in the box.

the interface. This situation is easily tackled in the network of Fig. 6 by just connecting, to each accessible mode, a new transmission line with the constitutive parameters of the new medium.

Once we have characterized the complete circuit with the network in Fig. 6, the S-parameters are computed by using one of the transverse port as excitation, and loading the other one with the reference impedance of the port $\left(Z_{0}=50 \Omega\right)$. The total matrix $\left(\overline{\mathbf{Z}}^{\mathbf{T}}\right)$ that models the complete problem can then be written in terms of the following voltage-current relation:

$\left[\begin{array}{c}V_{p 1} \\ 0 \\ 0 \\ \cdot \\ \cdot \\ 0\end{array}\right]=\left[\begin{array}{ccccc}Z_{p 1, p 1}^{T} & Z_{p 1, p 2}^{T} & Z_{p 1,1}^{T} & \cdot & Z_{p 1, N}^{T} \\ Z_{p 2, p 1}^{T} & Z_{p 2, p 2}^{T} & Z_{p 2,1}^{T} & \cdot & Z_{p 2, N}^{T} \\ Z_{1, p 1}^{T} & Z_{1, p 2}^{T} & Z_{1,1}^{T} & \cdot & Z_{1, N}^{T} \\ \cdot & \cdot & & \cdot & \cdot \\ \cdot & & \cdot & & \cdot \\ Z_{N, p 1}^{T} & Z_{N, p 2}^{T} & Z_{N, 1}^{T} & \cdot & Z_{N, N}^{T}\end{array}\right]\left[\begin{array}{c}I_{p 1} \\ I_{p 2} \\ I_{1}^{(1)} \\ \cdot \\ \cdot \\ I_{N}^{\prime}(2)\end{array}\right]$.

This system relates the external voltages $\left(V_{n}\right.$ and $\left.V_{n}^{\prime}\right)$ and the port voltages $\left(V_{p 1}\right.$ and $\left.V_{p 2}\right)$ in Fig. 6 to the external currents $\left(I_{n}^{(1)}\right.$ and $\left.I_{n}^{\prime(2)}\right)$ and the port currents $\left(I_{p 1}\right.$ and $\left.I_{p 2}\right)$. In (34), the external voltages have been replaced by their corresponding voltage-current relations. The input admittance $\left(Y_{\text {in }}=I_{p 1} / V_{p 1}\right)$ and the transadmittance between ports $\left(Y_{\text {tran }}=I_{p 2} / V_{p 1}\right)$ can then be extracted by solving the system in (34). The S-parameters are then calculated in terms of $Y_{\text {in }}$ and $Y_{\text {tran }}$ using again (32) and (33).

\section{Numerical Results AND ApPlications}

To validate the theoretical derivations, several examples of practical microwave printed circuits are analyzed in this section using the novel MEN formulation. The results are compared to the simulations obtained using other commercial software tools such as HFSS, other IE formulations [23] and real measurements from the prototypes.

The first example consists of a stepped-impedance lowpass filter implemented in microstrip technology, first presented in [31]. The structure of the filter under analysis is shown in Fig. 7. The dark areas correspond to the lumped ports. In Fig. 8 we show the filter response calculated with the MEN formulation proposed in this article. In the same figure, the

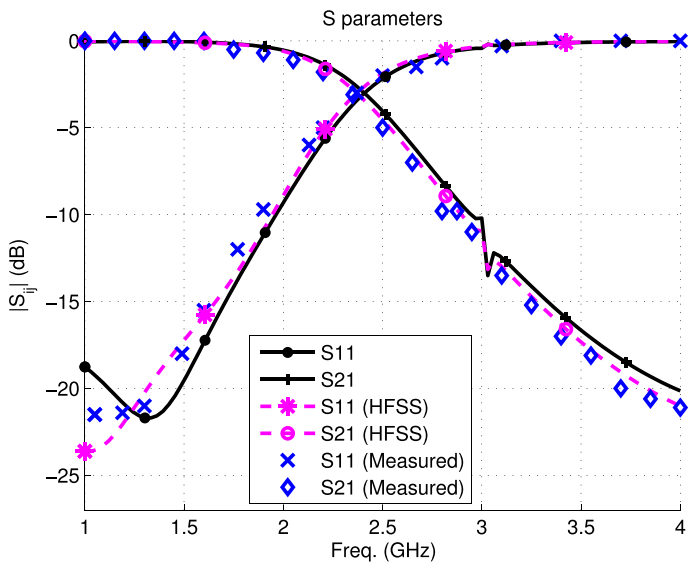

Fig. 8. Scattering parameters magnitude of the lowpass filter in Fig. 7 computed using the novel formulation, using a finite elements based electromagnetic analysis tool (HFSS), and compared to the measured response.

results obtained with a commercial software tool (HFSS) and the measured results are also reported.

As we can see, the MEN results are in good agreement with the response provided by HFSS, and also with measurements. To obtain this result, we have used 4000 terms in the kernel (3900 in the static part and 100 in the dynamic part of the kernel [28]). Furthermore, 600 basis and test functions (computed using the BI-RME method) have been used in the MoM-Galerkin procedure. Losses have not been included in this MEN simulation.

From the computational point of view, we first execute the BI-RME module to obtain the relevant coupling integrals and then run our MEN code. It is important to note that the static part of the kernel and the coupling integrals are not recomputed for each frequency point during the frequency sweep, since they are frequency-independent. For the results presented above, using an Intel (R) Core $^{(T M)}$ i7-6500U CPU at $2.50 \mathrm{GHz}$ with $12 \mathrm{~GB}$ of RAM, the computational time required to obtain the coupling integrals is less than $3 \mathrm{~min}$. The MEN code takes about $11.85 \mathrm{~s}$ for all the frequency sweep (100 points). The computational time of HFSS to obtain the results presented in this article is also given for completeness. In this case, HFSS takes 9 min and $3 \mathrm{~s}$ to obtain the response reported in Fig. 8.

The next example is presented in Fig. 9. This is a hairpin microstrip bandpass filter with two excitation ports in the transverse plane, originally proposed in [32]. Fig. 10 shows the in-band response obtained using our formulation. In this figure, the MEN results are compared to those obtained using the commercial software HFSS, and the simulated results from [32]. No losses are considered in any of the simulation results. For this example, we used 5000 terms in the kernel (4500 in the static part and 500 in the dynamic part of the kernel [28]) and 310 basis and test functions in the MoMGalerkin procedure. The computational time required for the MEN simulation is about $6.08 \mathrm{~s}$ (200 frequency points). It is important to mention that to obtain accurate results with HFSS, we need to use, in this case, a selectively refined mesh around the critical gaps. This increases to more than $30 \mathrm{~min}$ the 


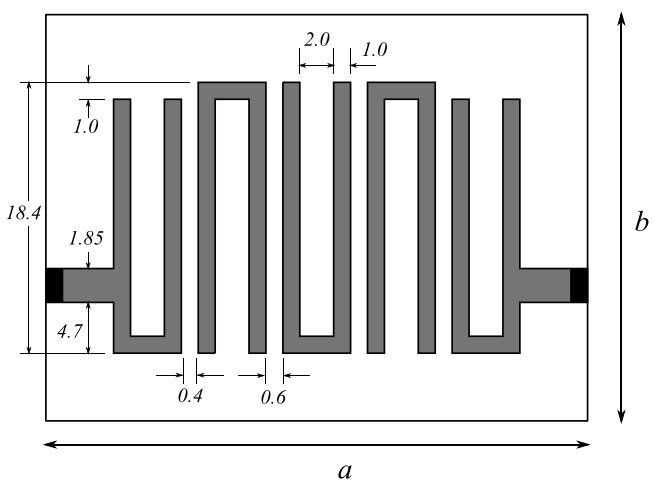

Fig. 9. Hairpin microstrip bandpass filter geometry under study. The dimensions are in $\mathrm{mm}$. The dimensions of the shielding box are: $a \times b=$ $31.2 \mathrm{~mm} \times 30 \mathrm{~mm}$. With respect to Fig. 3, the value of $l_{a}$ is $3.73 \mathrm{~mm}$. The dielectric relative permittivity is $\varepsilon_{r}=6.15$ and the substrate thickness is $l_{d}=1.27 \mathrm{~mm}$. The filter is centered in the box.

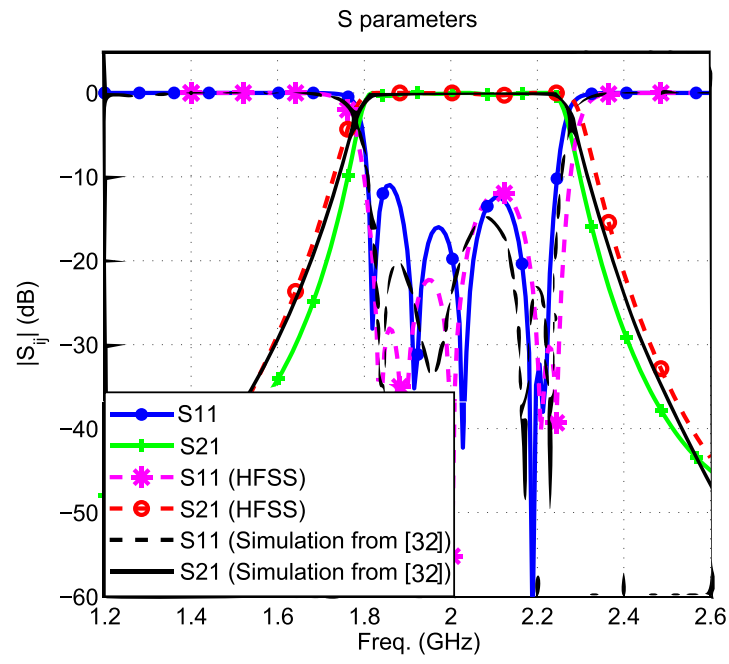

Fig. 10. In-band S-parameters magnitude of the hairpin bandpass filter shown in Fig. 9, using the proposed MEN formulation, and using both the electromagnetic analysis tool HFSS and results reported in [32].

computational time required by HFSS to obtain the results presented in Fig. 10. Additionally, Fig. 11 shows the outof-band response of this filter. As we can see, in both the in-band and out-of-band simulations, the MEN results show good agreement with respect to HFSS and the simulation data reported in [32].

The third example is the analysis of a dual-mode microstrip filter, first presented in [33]. The geometry of the microstrip circuit with the excitation port locations (dark areas) is shown in Fig. 12. In Fig. 13, we present the passband response obtained using our MEN formulation. In this case, losses are included in the MEN analysis. Details of the insertion losses in the passband are shown in the inset of Fig. 13, giving $\mathrm{IL}=$ $0.79 \mathrm{~dB}$. In particular, the following values have been used for modeling the losses: $\tan \delta=0.0047$ and $\sigma_{\text {box }}=3.8 \cdot 10^{7} \mathrm{~S} / \mathrm{m}$. The response obtained is compared to the HFSS response and also to the simulations and measurements reported in [33]. The simulations reported in [33] have been obtained using the commercially available full-wave electromagnetic simulator Sonnet. As it is reported in [33], all the simulations are normalized in the frequency axis using $f_{0}=1.07 \mathrm{GHz}$ (design

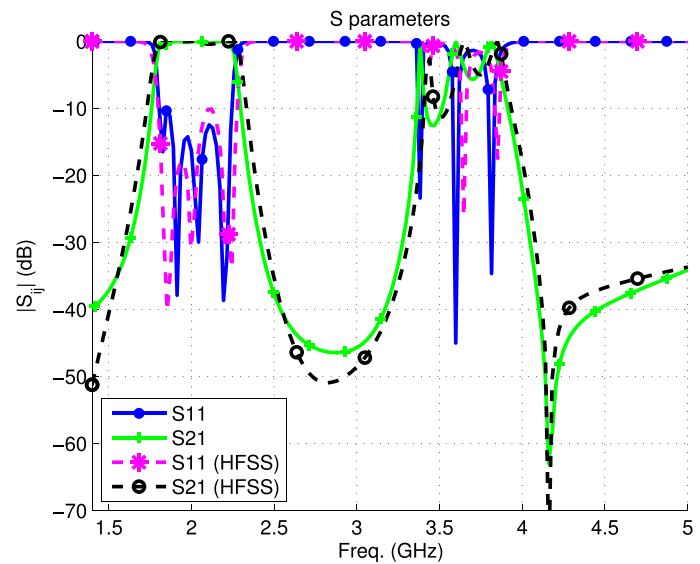

Fig. 11. Out-of-band S-parameters magnitude of the hairpin bandpass filter shown in Fig. 9, using the proposed MEN formulation and the electromagnetic analysis tool HFSS.

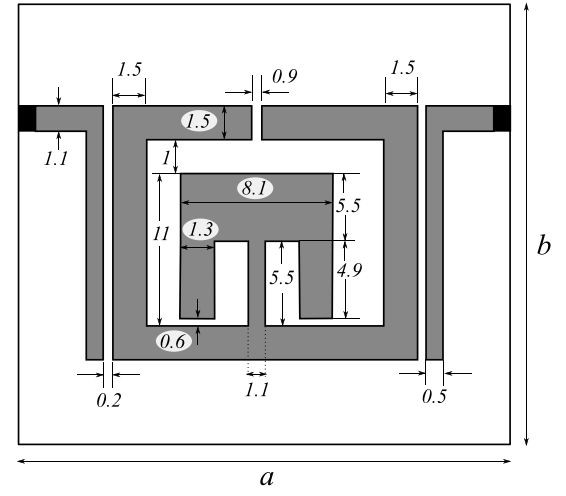

Fig. 12. Dual-mode microstrip bandpass filter under study. The dimensions are in $\mathrm{mm}$. The dimensions of the shielding box are: $a \times b=25 \mathrm{~mm} \times 20 \mathrm{~mm}$. With respect to Fig. 3, the value of $l_{a}$ is $8.73 \mathrm{~mm}$. The dielectric relative permittivity is $\varepsilon_{r}=10.8$ and the substrate thickness is $l_{d}=1.27 \mathrm{~mm}$. The filter is centered in the box.

center frequency) and the measurements are normalized using $f_{0}=0.9975 \mathrm{GHz}$ (measured center frequency).

To obtain the in-band response, 4000 terms have been used in the kernel (3700 in the static part and 300 in the dynamic part of the kernel [28]). In the MoM-Galerkin method, 450 basis and test functions (computed using the BI-RME method) have been used. The computational time required to obtain the coupling integrals is less than $6 \mathrm{~min}$. The MEN code takes about $11.41 \mathrm{~s}$ for the complete frequency sweep (120 points). To obtain the results presented in Fig. 13, HFSS takes $23 \mathrm{~min}$ and $6 \mathrm{~s}$. Finally, the out-of-band response is shown in Fig. 14, where the results obtained using the MEN technique are also compared to the HFSS response and the data from [33]. As we can see, the results are also in good agreement with respect to the theoretical and experimental data retrieved from [33].

The fourth example is the Wilkinson power divider shown in Fig. 15. This circuit has been chosen to validate the formulation for devices containing internal ports. In this circuit, the resistor $R$ is modeled as an internal port (hatched area) with reference impedance equal to $Z_{p}=R=2 Z_{0}=100 \Omega$. In addition, three external pulses (dark areas) are included to model the three external ports (Ports 1-3) of the power divider.

In Fig. 16, we show the response of the structure obtained using our MEN formulation and we compare it to the HFSS 


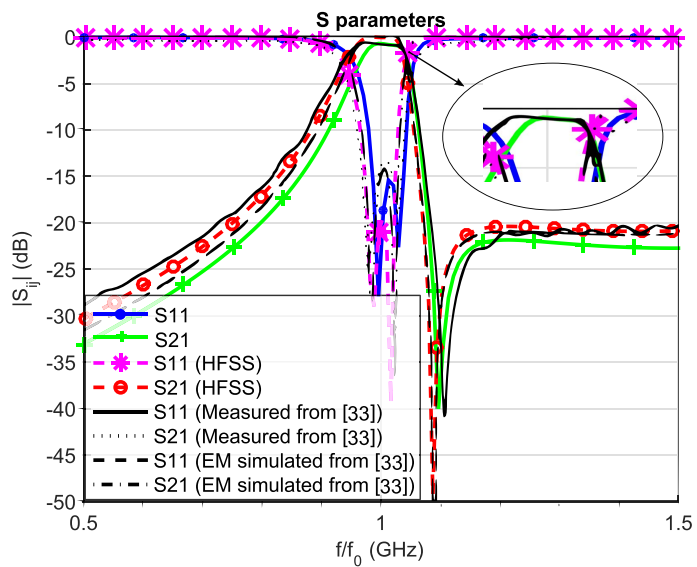

Fig. 13. In-band S-parameters magnitude of the dual-mode bandpass filter shown in Fig. 12, using the proposed lossy MEN formulation, and the electromagnetic analysis tool HFSS. Results of simulations (using Sonnet) and measurements from [33] are also included.

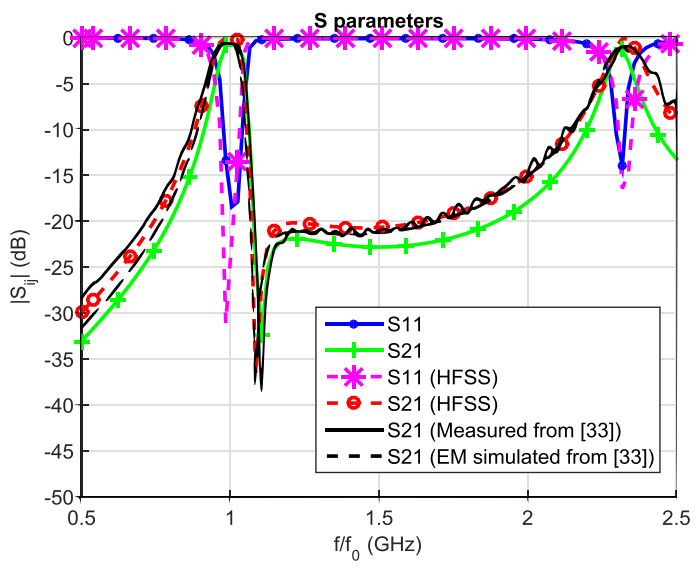

Fig. 14. Out-of-band S-parameters magnitude of the dual-mode bandpass filter shown in Fig. 12, using the proposed lossy MEN formulation, and the electromagnetic analysis tool HFSS. Results of simulations (using Sonnet), and measurements from [33] are also included.

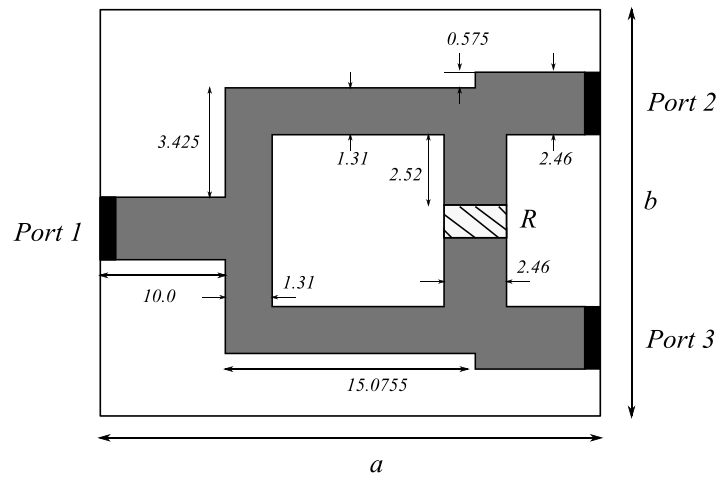

Fig. 15. Wilkinson power divider in microstrip technology. The dimensions are in $\mathrm{mm}$. The dimensions of the shielding box are: $a \times b=30 \mathrm{~mm} \times 16 \mathrm{~mm}$. The value of $l_{a}$ in Fig. 3 is $3 \mathrm{~mm}$. The dielectric relative permittivity is $\varepsilon_{r}=4$ and the substrate thickness is $l_{d}=1.2 \mathrm{~mm}$. The divider is centered in the box.

results when the divider is excited through Port 1. As we can see, the S-parameters obtained are in good agreement with the HFSS simulation. At the design frequency, half of the input power goes to Port $2\left(\left|S_{21}\right| \approx-3 \mathrm{~dB}\right)$ while the other half goes to Port $3\left(\left|S_{31}\right| \approx-3 \mathrm{~dB}\right)$. Port 1 exhibits good matching $\left(\left|S_{11}\right|<-20 \mathrm{~dB}\right)$. In this case, the resistor is not influencing the response. To further validate the internal

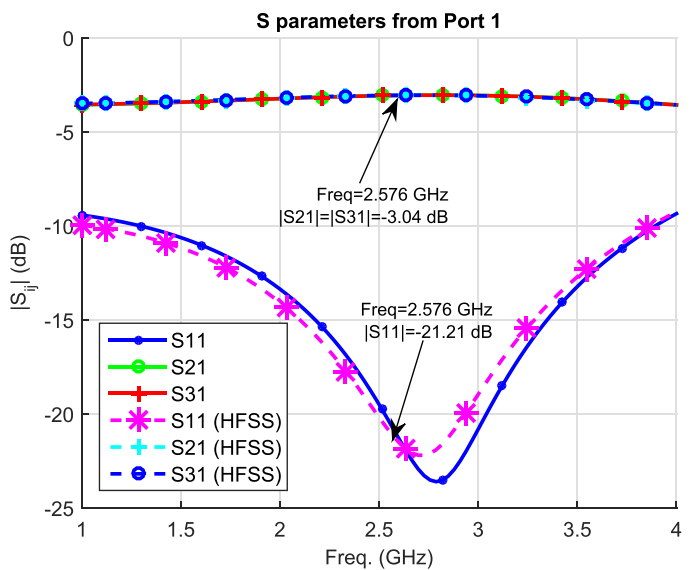

Fig. 16. S-parameters magnitude of the power divider, shown in Fig. 15, using the proposed MEN formulation and the commercial software HFSS. The excitation is through Port 1.

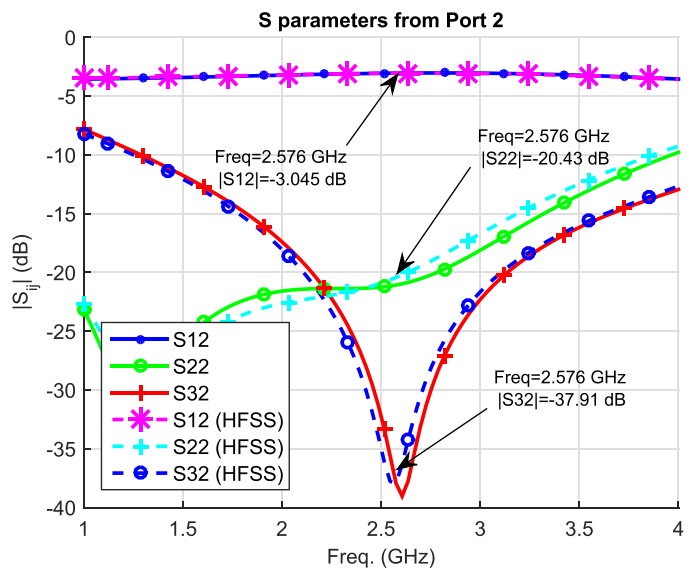

Fig. 17. S-parameters magnitude of the power divider, shown in Fig. 15, using the proposed MEN formulation and the commercial software HFSS. The excitation is through Port 2.

port performance, in Fig. 17 we show the response obtained using the MEN formulation and we compare it to the HFSS results, when the structure is excited through Port 2. As we can see, good agreement between our results and the HFSS results is obtained also in this case. From Fig. 17, we can verify the good performance of the internal port (used to connect the resistor), since half of the power goes from Port 2 to Port $1\left(\left|S_{12}\right| \approx-3 \mathrm{~dB}\right)$ and Port 3 remains isolated $\left(\left|S_{32}\right|<-37 \mathrm{~dB}\right)$. Additionally, the resistor dissipates half of the power, since Port 2 remains with good matching levels $\left(\left|S_{22}\right|<-20 \mathrm{~dB}\right)$.

In the last test, we show how the internal port behaves when the value of the resistor is changed from the ideal nominal value of $R=100 \Omega$, to a much lower value $R=10 \Omega$. As we can see from Fig. 18, in this situation, Port 3 is not isolated anymore, and Port 2 exhibits poor matching. Again, the MEN results are in good agreement with the commercial software tool HFSS. In particular, our formulation correctly predicts a drop in the matching level of Port 2 and in the isolation to Port 3, giving $\left|S_{22}\right|=\left|S_{32}\right|=-7.61 \mathrm{~dB}$ at the design frequency. The same values are also predicted by the commercial tool HFSS.

To obtain the responses reported in Figs. 16-18, 4000 terms have been used in the kernel (3900 in the static part and 100 


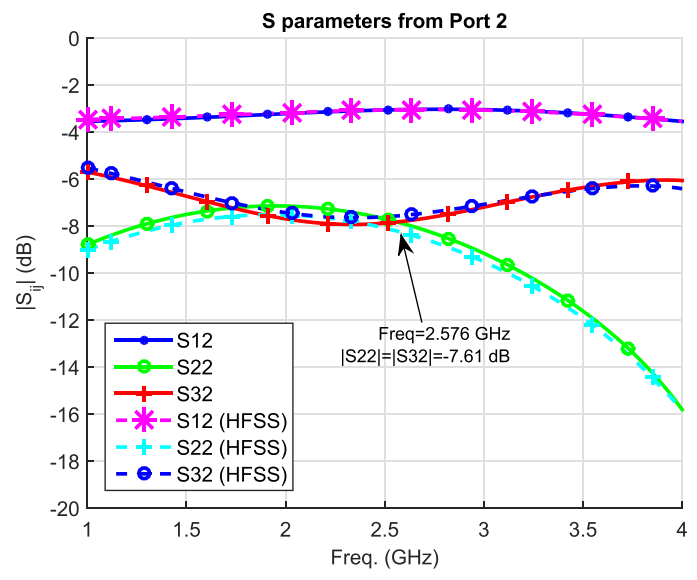

Fig. 18. S-parameters magnitude of the power divider, shown in Fig. 15, when the internal resistor has $R=10 \Omega$. The simulations are obtained using the MEN formulation and the commercial software HFSS. The excitation is through Port 2.

in the dynamic part of the kernel [28]). In the MoM-Galerkin procedure, 350 basis and test functions (computed using the BI-RME method) have been used. The computational time required to obtain the coupling integrals is less than 4 min. The MEN code takes about $4.54 \mathrm{~s}$ to obtain the results in Figs. 16 and 17 (for 100 frequency points) and the same computational time for the results in Fig. 18. The coupling integrals do not have to be recomputed for the second case $(R=10 \Omega)$ since the metallization has not changed as compared to the first case $(R=100 \Omega)$. HFSS takes about 10 min to obtain the results in Figs. 16 and 17 and the same time to obtain the results in Fig. 18.

It is important to mention that, for the four examples discussed so far, only one accessible mode is required to solve the problem, since all structures contain only one discontinuity in the transverse plane (only one metallic circuit, including the two input and output ports). Besides the advantages discussed in this article, we should remark that the analysis of structures with very thin dielectric layers and several metallization interfaces in close proximity, will require more computational effort. In the case of the MEN technique discussed in this article, this situation will require the use of more accessible modes in the equivalent network, to correctly characterize the energy exchange process between closely spaced discontinuities. The use of more accessible modes will increase the size of the final matrix, and consequently the time required to solve large systems of linear equations will be also higher. This increase in computational time will be evident in the examples that are discussed next.

The last example consists of a microwave resonator with direct input and output coupling, implemented through a multilayer stack as shown in Fig. 5. The circuit is composed of three dielectric layers and two metallic interfaces (the microstrip circuits). In this section, we discuss three different implementations of this filter. The first implementation is based on rectangular metallizations. The layout of the two metallic interfaces is shown in Fig. 19. The first (lower) level contains the input and output microstrip lines. The second level contains a rectangular microstrip resonator. This multilayer implementation allows for a direct capacitive coupling
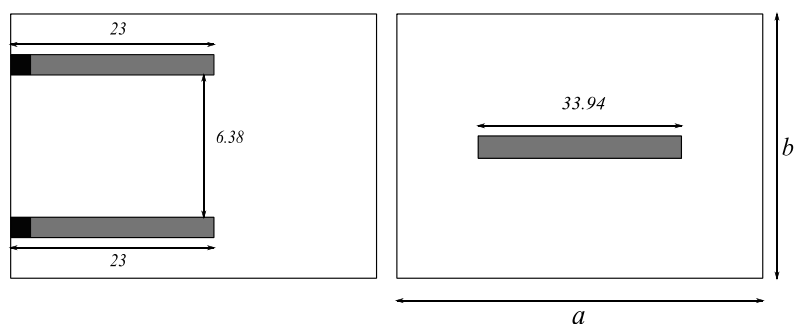

Fig. 19. First implementation of a microwave resonator with direct input/output coupling, using two metallization interfaces. The dimensions are in $\mathrm{mm}$. Gray areas are the metallizations while dark areas represent the excitation ports. The width of all lines is $1.49 \mathrm{~mm}$. The dimensions of the shielding box are $a \times b=40 \mathrm{~mm} \times 20 \mathrm{~mm}$. With respect to Fig. 5, the heights of the dielectric layers are: $h_{1}=h_{2}=h_{3}=5 \mathrm{~mm}$. The relative dielectric permittivities of the substrates are: $\varepsilon_{r 1}=\varepsilon_{r 3}=2.33$ and $\varepsilon_{r 2}=1.07$.

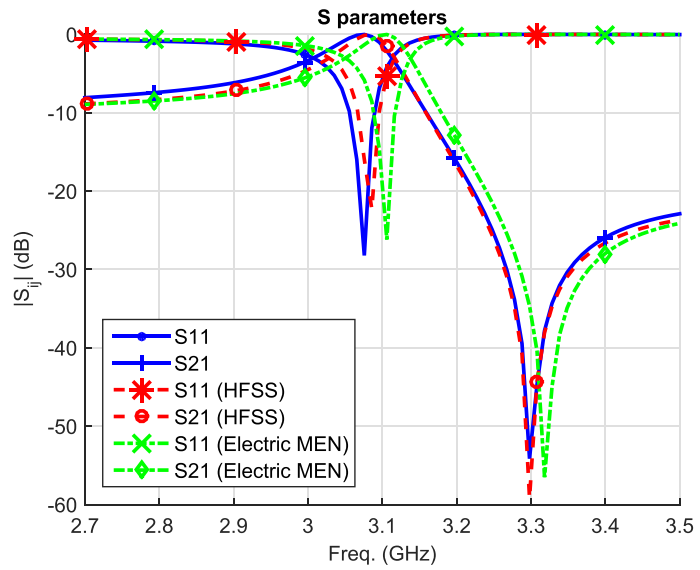

Fig. 20. S-parameter magnitude of the rectangular microwave resonator of Fig. 19, obtained using the our formulation, compared with the results obtained using the formulation reported in [23], and to the simulation results obtained with HFSS.

between input and output ports ( $P 1-P 2$ coupling), so that one transmission zero (TZ) appears above the passband. Again, the structure is completely shielded, and the excitations are the two lateral coaxial connectors (see Fig. 5) that are modeled with pulse ports (dark areas in Fig. 19).

The performance of the filter, obtained using our MEN formulation, is presented in Fig. 20. In this figure, we compare the results with the full-wave simulation using the electric MEN formulation reported in [23], and the results from HFSS. As we can see, the simulated S-parameters are in good agreement with the results obtained using the commercial software HFSS. Also, the response is similar to the one obtained using the electric multilayer MEN formulation described in [23], although with a slight frequency shift.

Another possible implementation of the structure is using an elliptical resonator, as shown in Fig. 21. In this case, since the resonator is not rectangular, the analysis cannot be performed using the electric MEN technique described in [22] and [23] or the direct EFIE formulation proposed in [7]. However, this analysis can still be carried out using the MEN formulation proposed in this article. The MEN results are compared to HFSS simulations in Fig. 22. As we can see, good agreement between the MEN simulation and HFSS results is obtained also in this case. The resonator in Fig. 21 has been tuned so that its response is similar to the rectangular resonator of Fig. 19. 
TABLE I

Numerical Parameters and Computational Time Required by All the Microwave Components Analyzed in This Article. $N$ is the Number of Accessible Modes and $N_{b}$ Represents the Number of Basis/Test Functions Used in the MoM-Galerkin Procedure.

\begin{tabular}{|c|c|c|c|c|c|c|}
\hline Device & $N$ & $N_{b}$ & Terms in dynamic / static kernel & Num. of frequency points & BI-RME time & MEN time \\
\hline \hline Lowpass filter & 1 & 600 & $100 / 3900$ & 100 & $3 \mathrm{~min}$ & $11.85 \mathrm{~s}$ \\
Hairpin filter & 1 & 310 & $500 / 4500$ & 200 & $4 \mathrm{~min}$ & $6.08 \mathrm{~s}$ \\
Dual-mode filter & 1 & 450 & $300 / 3700$ & 120 & $6 \mathrm{~min}$ & $11.41 \mathrm{~s}$ \\
Wilkinson divider & 1 & 350 & $100 / 3900$ & 100 & $4 \mathrm{~min}$ & $4.54 \mathrm{~s}$ \\
Multilayer filters & 30 & 400 & $300 / 3700$ & 100 & $3 \mathrm{~min}+3 \mathrm{~min}$ & $1 \mathrm{~min} 40 \mathrm{~s}$ \\
\hline
\end{tabular}
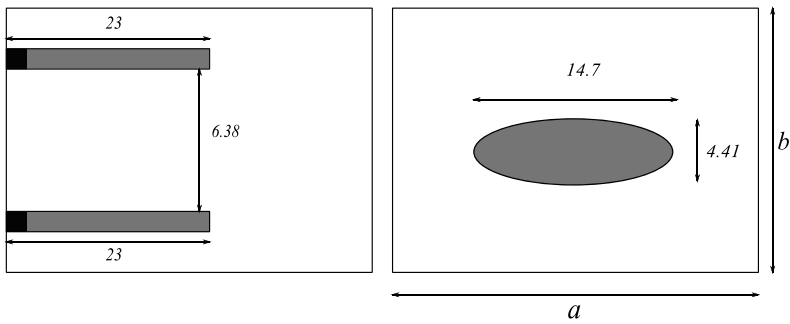

Fig. 21. Second implementation of a microwave resonator with direct input/output coupling, using two metallizations. In this case, an elliptic resonator is used in the second metallization level. The dimensions are in $\mathrm{mm}$. The width of the rectangular input/output lines is $1.49 \mathrm{~mm}$. The dimensions of the shielding box are $a \times b=40 \mathrm{~mm} \times 20 \mathrm{~mm}$. With respect to Fig. 5, $h_{1}=h_{3}=5 \mathrm{~mm}$ and $h_{2}=6.52 \mathrm{~mm}$. The relative dielectric permittivities of the substrates are $\varepsilon_{r 1}=2.33, \varepsilon_{r 2}=1.07$, and $\varepsilon_{r 3}=4$.

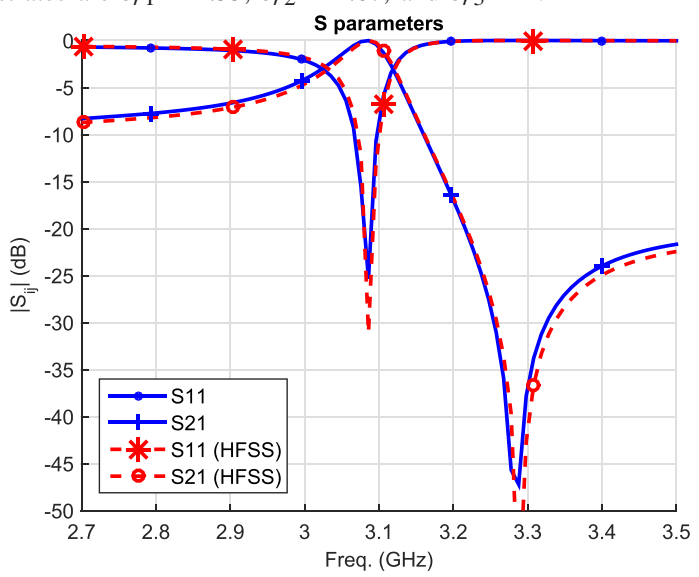

Fig. 22. S-parameter magnitude of the elliptic microwave resonator of Fig. 21, obtained using the presented formulation and compared to the simulation results obtained with the commercial tool HFSS.

As discussed before, these two implementations have a TZ above the passband due to the direct capacitive coupling between input and output ports $(P 1-P 2)$. If the $\mathrm{TZ}$ is required to be below the passband, the sign of the direct coupling $P 1-P 2$ should be changed. This can be implemented through a direct inductive coupling between the ports.

The third design shows a practical implementation of this concept (see Fig. 23). Here, we propose to use an inductor $L$ (connected to an internal port) between the input and the output lines to change the sign of the direct $P 1-P 2$ coupling. Fig. 24 shows the response obtained using our formulation. In this figure, the MEN results are compared to those obtained using the commercial software HFSS. As we can see, the MEN simulation is in good agreement with respect to the analysis performed using the commercial software HFSS. As expected, with the inductance, the TZ is shifted below the passband. The dimensions of the filter, given in Fig. 23, have been tuned for the same center frequency as for the microwave resonators in
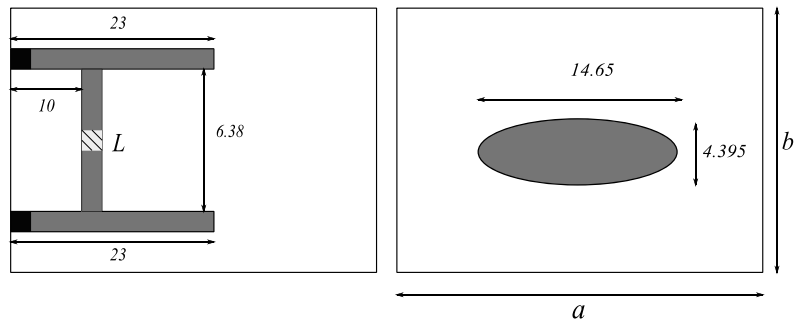

Fig. 23. Third implementation of a microwave resonator with direct input/output coupling, using two metallization interfaces. An internal port is used to introduce a direct inductive coupling between input/output ports. The dimensions are in $\mathrm{mm}$. The width of the input/output lines is $1.49 \mathrm{~mm}$. The internal port (hatched area) is used to connect an inductor $L$. The dimensions of the shielding box are $a \times b=40 \mathrm{~mm} \times 20 \mathrm{~mm}$. With respect to Fig. 5, $h_{1}=h_{3}=5 \mathrm{~mm}$ and $h_{2}=7.5 \mathrm{~mm}$. The relative dielectric permittivities of the substrates are the same as in Fig. 21. The value of the inductor is $L=25 \mathrm{nH}$.

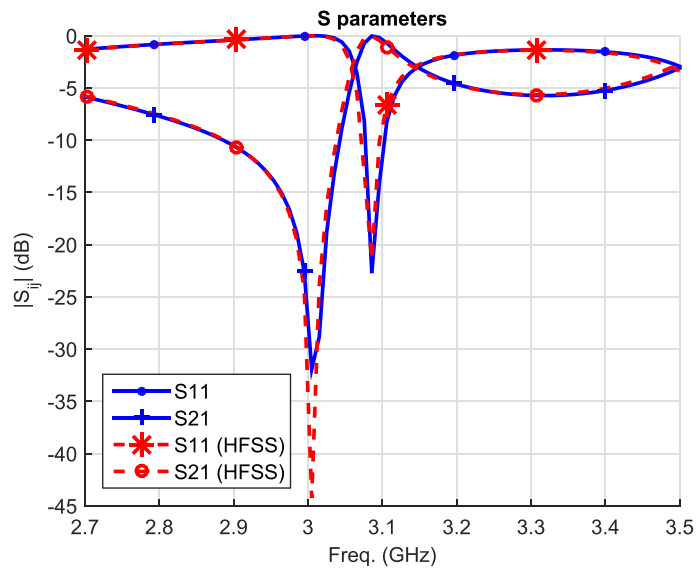

Fig. 24. S-parameters magnitude of the elliptic microwave resonator with inductive input/output coupling, as shown in Fig. 23, using the proposed MEN formulation and the software tool HFSS.

Figs. 19 and 21. This example further validates the treatment of internal ports presented in this article.

For these simulations, we have used 30 accessible modes for the equivalent network of Fig. 6. To characterize the two metallic interfaces, we have included 400 basis and test functions in the MoM-Galerkin procedure, 3700 terms in the static kernel, and 300 in the dynamic kernel. To analyze the complete structure, the computational time required by the MEN code for each design is about $1 \mathrm{~min}$ and $40 \mathrm{~s}$ (for 100 points in the frequency sweep). The computational time required to obtain the coupling integrals is less than $3 \mathrm{~min}$. For each simulation of this last example, HFSS takes about 13 min using the same frequency sweep.

Finally, we summarize in Table I the simulation data for all the examples analyzed using the MEN. With the examples discussed in this section, the theory proposed for the 
rigorous analysis of zero-thickness printed circuits composed of metallizations with arbitrary shape that can contain internal and external ports has been fully validated. Structures that contain more than one metallic level can also be efficiently analyzed. These results also confirm once more that, although other numerical methods are much more powerful from the point of view of the structures they can analyze, if a modal analysis formulation can be obtained, the computational time required to perform the electromagnetic analysis is significantly reduced. In conclusion, in this section, we have demonstrated that the MEN approach described in this article for analysis of practical single-layer and multilayer shielded printed circuits is both efficient and accurate.

\section{CONCLUSION}

In this article, the applicability of the rigorous MEN technique has been extended to the analysis of planar circuits (or discontinuities) that include arbitrarily shaped metalizations together with internal and external ports in the transverse plane. Using the reported novel formulation, a very large variety of practical single-layer and multilayer boxed microwave printed circuits can be efficiently analyzed in the common frame of the MEN technique. The inclusion of losses in the structure under analysis has also been discussed. In addition to theory, the analysis of a number of practical planar structures has been performed, showing good agreement with respect to other electromagnetic analysis tools, and with measured results. The examples discussed fully validate the theoretical formulation that we propose, and clearly confirm the usefulness and efficiency of the novel procedure in the analysis of real shielded planar microstrip circuits.

\section{REFERENCES}

[1] T. Itoh, Numerical Techniques for Microwave and Millimeter-Wave Passive Structures. Hoboken, NJ, USA: Wiley, 1989.

[2] J.-M. Jin, Theory and Computation of Electromagnetic Fields Hoboken, NJ, USA: Wiley, 2015.

[3] M. Salazar Palma, T. K. Sarkar, L. E. Garcia Castillo, T. Roy, and A. Djordjevic, Iterative and Self-Adaptive Finite Elements in Electromagnetic Modeling. Norwood, MA, USA: Artech House, Inc., 1998.

[4] A. F. Peterson, S. L. Ray, and R. Mittra, Finite-Difference Time Domain Methods Orthogonal Meshes. Hoboken, NJ, USA: Wiley, 1998, pp. 495-523.

[5] P. Arcioni, M. Bressan, G. Conciauro, and L. Perregrini, "Generalized Y-matrix of arbitrary H-plane waveguide junctions by the BI-RME method," in IEEE MTT-S Int. Microw. Symp. Dig., Denver, CO, USA, Jun. 1997, pp. 211-214.

[6] A. Constantine Balanis, Advanced Engineering Electromagnetics. Hoboken, NJ, USA: Wiley, 2012.

[7] A. A. Melcon, J. R. Mosig, and M. Guglielmi, "Efficient CAD of boxed microwave circuits based on arbitrary rectangular elements," IEEE Trans. Microw. Theory Techn., vol. 47, no. 7, pp. 1045-1058, Jul. 1999.

[8] M. Bozzi, L. Perregrini, A. Á. Melcón, M. Guglielmi, and G. Conciauro, "MoM/BI-RME analysis of boxed MMICs with arbitrarily shaped metallizations," IEEE Trans. Microw. Theory Techn., vol. 49, no. 12, pp. 2227-2234, Dec. 2001.

[9] J.C. Rautio and R.F. Harrington, "An electromagnetic time-harmonic analysis of shielded microstrip circuits," IEEE Trans. Microw. Theory Techn., vol. 35, no. 8, pp. 726-730, Aug. 1987.

[10] I. Park, R. Mittra, and M. I. Aksun, "Numerically efficient analysis of planar microstrip configurations using closed-form Green's functions," IEEE Trans. Microw. Theory Techn., vol. 43, no. 2, pp. 394-400, Feb. 1995.

[11] N. K. and M. I. Aksun "Efficient use of closed-form Green's functions for the analysis of planar geometries with vertical connections," IEEE Trans. Microw. Theory Techn., vol. 45, no. 5, pp. 593-603, May 1997.
[12] M. A. Marin, S. Barkeshli, and P. H. Pathak, "Efficient analysis of planar microstrip geometries using a closed-form asymptotic representation of the grounded dielectric slab Green's function," IEEE Trans. Microw. Theory Techn., vol. 37, no. 4, pp. 669-679, Apr. 1989.

[13] S. G. Pan and I. Wolff, "Scalarization of dyadic spectral Green's functions and network formalism for three-dimensional full-wave analysis of planar lines and antennas," IEEE Trans. Microw. Theory Techn., vol. 42, no. 11, pp. 2118-2127, Nov. 1994.

[14] T. M. Grzegorczyk and J. R. Mosig, "Full-wave analysis of antennas containing horizontal and vertical metallizations embedded in planar multilayered media," IEEE Trans. Antennas Prop., vol. 51, no. 11, pp. 3047-3054, Nov. 2003.

[15] G. Conciauro, M. Guglielmi, and R. Sorrentino, Advanced Modal Analysis: CAD Techniques for Waveguide Components and Filter. New York, NY, USA: Wiley, 1999.

[16] N. Marcuvitz, Waveguide Handbook (MIT Radiation Laboratory Series), vol. 10. New York, NY, USA: McGraw-Hill, 1951.

[17] H. K. Smith, J. R. Mosig, and M. Guglielmi, "Multimode network approach for the solution of two-dimensional waveguide discontinuities," in Proc. 23rd Eur. Microw. Conf., Madrid, Spain, Sep. 1993, pp. $670-672$.

[18] A. A. Melcon and M. Guglielmi, "Multimode network representation of two dimensional steps in rectangular waveguides," in Proc. 24th Eur. Microw. Conf., Cannes, France, Sep. 1994, pp. 1943-1948.

[19] G. Gerini, M. Guglielmi, and G. Lastoria, "Efficient integral equation formulations for admittance or impedance representation of planar waveguide junctions," in IEEE MTT-S Int. Microw. Symp. Dig., Baltimore, MD, USA, Jun. 1998, pp. 1747-1750.

[20] M. Guglielmi and A. A. Oliner, "Multimode network description of a planar periodic metal-strip grating at a dielectric interface. I. Rigorous network formulations," IEEE Trans. Microw. Theory Techn., vol. 37, no. 3, pp. 534-541, Mar. 1989.

[21] S. Monni, G. Gerini, A. Neto, and A. G. Tijhuis, "Multimode equivalent networks for the design and analysis of frequency selective surfaces," IEEE Trans. Antennas Propag., vol. 55, no. 10, pp. 2824-2835, Oct. 2007.

[22] C. G. Molina et al., "Rigorous multimode equivalent network representation of multilayer planar circuits," in IEEE MTT-S Int. Microw. Symp. Dig., Aug. 2018, pp. 1-4.

[23] C. G. Molina, F. Q. Pereira, A. Á. Melcón, V. E. Boria, and M. Guglielmi, "Electric multimode equivalent network technique for multilayer shielded circuits based on arbitrary rectangular elements," in Proc. Int. Conf. Electromagn. Adv. Appl. (ICEAA), Sep. 2019, pp. 1043-1047.

[24] G. Conciauro and M. C. A. Bressan Zuffada "Waveguide modes via an integral equation leading to a linear matrix eigenvalue problem," IEEE Trans. Microw. Theory Techn., vol. 32, no. 11, pp. 1495-1504, Nov. 1984.

[25] S. Cogollos, S. Marini, V. E. Boria, P. Soto, A. Vidal, H. Esteban, J. V. Morro, and B. Gimeno, "Efficient modal analysis of arbitrarily shaped waveguides composed of linear, circular, and elliptical arcs using the BI-RME method," IEEE Trans. Microw. Theory Techn., vol. 51, no. 12, pp. 2378-2390, Dec. 2003.

[26] G. V. Eleftheriades and J. R. Mosig, "On the network characterization of planar passive circuits using the method of moments," IEEE Trans. Microw. Theory Techn., vol. 44, no. 3, pp. 438-445, Mar. 1996.

[27] C. Gómez Molina, F. Quesada Pereira, A. Á. Melcón, V. E. Boria, and M. Guglielmi, "An efficient technique to assess the convergence of the multimode equivalent network for waveguide devices," IEEE Trans. Microw. Theory Techn., vol. 66, no. 2, pp. 651-658, Feb. 2018.

[28] C. G. Molina, F. Q. Pereira, A. A. Melcon, V. B. Esbert, and M. Guglielmi, "Efficient formulation of multimode equivalent networks for 2-D waveguide steps through Kummer's transformation," in IEEE MTT-S Int. Microw. Symp. Dig., May 2017.

[29] J. C. Rautio, and V. Demir, "Microstrip conductor loss models for electromagnetic analysis," IEEE Trans. Microw. Theory Techn., vol. 51, no. 3, pp. 915-921, Mar. 2003.

[30] M. Taroncher, J. Hueso, S. Cogollos, B. Gimeno, V. E. Boria, A. Vidal, H. Esteban, and M. Guglielmi, "Accurate consideration of metal losses at waveguide junctions using admittance and impedance integral equation formulations," Radio Sci., vol. 40, no. 6, 2005, Art. no. RS6013.

[31] G. V. Eleftherlades, J. R. Mosig, and M. Guglielmi, "An efficient mixed potential integral equation technique for the analysis of shielded MMIC's," in Proc. 25th Eur. Microw. Conf., Bologna, Italy, Sep. 1995, pp. 825-829. 
[32] J. S. Hong and M. J. Lancaster, Microstrip Filters for RF/Microwave Applications. Hoboken, NJ, USA: Wiley, 2001.

[33] J. S. Hong, H. Shaman, and Y.-H. Chun, "Dual-mode microstrip openloop resonators and filters," IEEE Trans. Microw. Theory Techn., vol. 55 , no. 8, pp. 1764-1770, Aug. 2007.

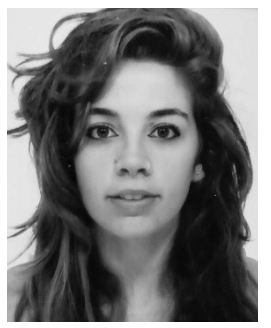

Celia Gómez Molina was born in Murcia, Spain, in 1992. She received the degree in telecommunication systems from the Technical University of Cartagena (UPCT), Murcia, in 2014, and the master's degree in telecommunication engineering from the Technical University of Cartagena (UPCT), Murcia, in 2016.

In 2016, she joined the Department of Information Technologies and Communications, UPCT, as a Research Student, where she is currently developing her teaching and research activities. She was a Visiting Student with the University of California, Davis, CA, USA, from April 2, 2018, to June 22, 2018, and the Politecnico di Milano, Milan, Italy, from April 1, 2019, to June 28, 2019. She is also involved in the development of analytical and numerical tools for network representations of waveguide and planar discontinuities. Her current scientific interest includes the design of microwave filters using different technologies.

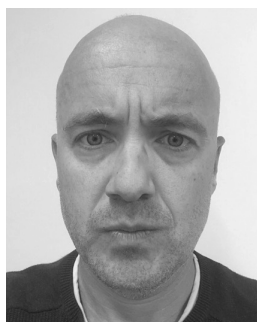

Fernando Quesada Pereira (Member, IEEE) was born in Murcia, Spain, in 1974. He received the master's degree in telecommunications engineering from the Technical University of Valencia (UPV), Valencia, Spain, in 2000, and the Ph.D. degree from the Technical University of Cartagena (UPCT), Murcia, in 2007.

In 1999, he joined the Radiocommunications Department, UPV, as a Research Assistant, where he was involved in the development of numerical methods for the analysis of anechoic chambers and tag antennas. In 2001, he joined the Communications and Information Technologies Department, UPCT, as a Research Assistant, and then as an Assistant Professor. In 2005, he was a Visiting Scientist with the University of Pavia, Pavia, Italy. In 2009, he was an Invited Researcher with UPV. In 2011, he became an Associate Professor with UPCT. His current research interest includes integral equation numerical methods for the analysis of antennas and microwave devices, along with microwave filters design and applications.

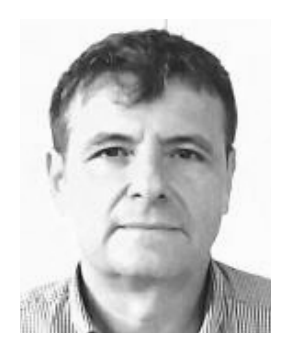

Alejandro Álvarez Melcón (Senior Member, IEEE) was born in Madrid, Spain, in 1965. He received the master's degree in telecommunications engineering from the Technical University of Madrid (UPM), Madrid, Spain, in 1991, and the Ph.D. degree in electrical engineering from the Swiss Federal Institute of Technology, Lausanne, Switzerland, in 1998.

In 1988, he joined the Signal, Systems, and Radiocommunications Department, UPM, as a Research Student, where he was involved in the design, testing, and measurement of broad-band spiral antennas for electromagnetic measurements support (EMS) equipment. From 1991 to 1993, he was with the Radio Frequency Systems Division, European Space Agency (ESA/ESTEC), Noordwijk, The Netherlands, where he was also involved in the development of analytical and numerical tools for the study of waveguide discontinuities, planar transmission lines, and microwave filters. From 1993 to 1995 , he was with the Space Division, Industry Alcatel Espacio, Madrid, and was also with ESA, where he collaborated in several ESA/European Space Research and Technology Centre (ESTEC) contracts. From 1995 to 1999, he was with the Swiss Federal Institute of Technology, École Polytechnique Fédérale de Lausanne (EPFL), where he was involved with the field of microstrip antennas and printed circuits for space applications. In 2000, he joined the Technical University of Cartagena, Cartagena, Spain, where he is currently developing his teaching and research activities. $\mathrm{He}$ was an Invited Professor with the University of Montreal, Montreal, QC, Canada, from July 1, 2010 to September 30, 2010, and as a Visiting Professor with the University of California, Davis, CA, USA, from October 2, 2017 to September 27, 2018.

Dr. Alvarez Melcón was a recipient of the Journée Internationales de Nice Sur les Antennes (JINA) Best Paper Award for the best contribution to the JINA'98 International Symposium on Antennas, and the Colegio Oficial de Ingenieros de Telecomunicación (COIT/AEIT) Award to the best Ph.D. dissertation in basic information and communication technologies.

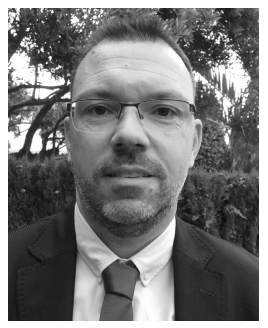

Stephan Marini (Senior Member, IEEE) received the Laurea degree in electronics engineering from the University of Perugia, Perugia, Italy, in 2001, and the Ph.D. degree in telecommunications from the Universidad Politécnica de Valencia, Valencia, Spain, in 2005

In 2001, he joined the Departamento de Comunicaciones, Universidad Politécnica de Valencia, where he was involved in the framework of the European Union Project-Millimeter-Wave and Microwave Components Design Framework for Ground and Space Multimedia Network (MMCODEF). Since 2005, he has been a Lecturer with the Departamento de Física, Ingeniería de Sistemas y Teoría de la Señal, Universidad de Alicante, Alicante, Spain, where he became an Associate Professor in 2012. In 2009, he received a fellowship from the Spanish Government for a short stay at the LEMA Laboratory, École Polytechnique Fédérale de Lausanne, Lausanne, Switzerland, as a Visiting Scientist. His current research interests include numerical methods in computer-aided techniques for the analysis and design of microwave and millimeter passive components and simulation of power effects (multipactor and corona) in passive waveguide systems.

Dr. Marini currently serves as an Associate Editor for IET Electronics Letters and as a regular Reviewer for the most relevant IEEE and IET technical journals in his areas of interest.

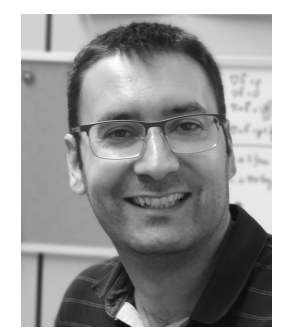

Miguel Ángel Sánchez-Soriano (Senior Member, IEEE) was born in Murcia, Spain, in 1984. He received the master's degree in telecommunications engineering (with a Special Award) and the Ph.D. degree in electrical engineering from Miguel Hernandez University (UMH), Elche, Spain, in 2007 and 2012, respectively.

In 2007, he joined the Radiofrequency Systems Group, UMH, as a Research Assistant. In 2010, he was a Visiting Researcher with the Microwaves Group, Heriot-Watt University, Edinburgh, U.K. In January 2013, he joined the LabSTICC Group, Université de Bretagne Occidentale, Brest, France, as a Post-Doctoral Researcher, where he worked for two years. From January 2015 to September 2015, he was a "Juan de la Cierva" Research Fellow with the Grupo de Aplicaciones de Microondas (GAM), Technical University of Valencia, Valencia, Spain. Since September 2015, he has been with the University of Alicante, Alicante, Spain, where he is currently an Associate Professor. His research interests cover the analysis and design of microwave planar devices, especially filters and their reconfigurability, and the multiphysics study of high frequency devices.

Dr. Sanchez-Soriano was a recipient of the runner-up HISPASAT Award to the Best Spanish Doctoral Thesis in New Applications for Satellite Communications, awarded by the Spanish Telecommunication Engineers Association (COIT/AEIT) and of the Extraordinary Ph.D. Award from Miguel Hernández University. He serves as an Associate Editor for the journals, IEEE TRANSACTIONS ON MiCROWAVES, THEORY AND TECHNIQUES and IET Microwaves, Antennas and Propagation and as a regular reviewer for more than 20 journals, including the IEEE MICROWAVE AND WIRELESS COMPONENTS LETTERS, IET Electronics Letters, IEEE ACCESS, and several IEEE international conferences. 


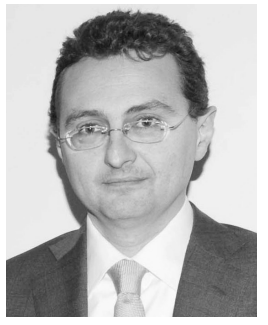

Vicente E. Boria (Fellow, IEEE) was born in Valencia, Spain, in May 18, 1970. He received the master's degree in Ingeniero de Telecomunicación (Hons.) and the Ph.D. degree in Ingeniero de Telecomunicación from the Universidad Politécnica de Valencia, Valencia, in 1993 and 1997, respectively.

In 1993, he joined the Departamento de Comunicaciones, Universidad Politécnica de Valencia, where he has been a Full Professor since 2003. In 1995 and 1996, he was holding a Spanish Trainee position at the European Space Research and Technology Centre, European Space Agency (ESTEC-ESA), Noordwijk, The Netherlands, where he was involved in the area of electromagnetics (EM) analysis and design of passive waveguide devices. He has authored or coauthored 15 chapters in technical textbooks, 180 articles in refereed international technical journals, and over 200 papers in international conference proceedings. His current research interests include the analysis and automated design of passive components (in particular filters and multiplexers) in several technologies, as well as on the simulation and measurement of power effects in highfrequency devices and systems.

Dr. Boria has been a member of the IEEE Microwave Theory and Techniques Society (IEEE MTT-S) and the IEEE Antennas and Propagation Society (IEEE AP-S) since 1992. He is also member of the Technical Committees of the IEEE-MTT International Microwave Symposium and the European Microwave Conference. He is also member of the European Microwave Association (EuMA) and has been the Chair of the 48th European Microwave Conference held in Madrid, Spain. He acts as a regular reviewer of the most relevant IEEE and IET technical journals on his areas of interest. He has been an Associate Editor of the IEEE MiCROWAVE AND WIRELESS COMPONENTS LETTERS from 2013 to 2018 and IET Electronics Letters from 2015 to 2018. He currently serves as a Subject Editor (Microwaves) of IET Electronics Letters, and as an Editorial Board Member of the International Journal of RF and Microwave Computer-Aided Engineering.

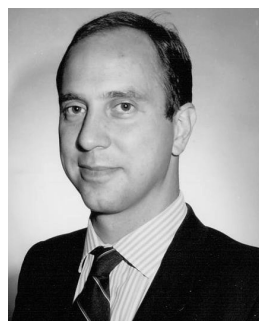

Marco Guglielmi (Life Fellow, IEEE) was born in Rome, Italy, in December 17, 1954. He received the Laurea degree in Ingegneria Elettronica from the University of Rome "La Sapienza", Rome, in 1979, where in 1980, he also attended the "Scuola di Specializzazione in Elettromagnetismo Applicato," the M.S. degree in electrical engineering from the University of Bridgeport, Bridgeport, CT, USA, in 1982, and the Ph.D. degree in electrophysics from Polytechnic University, Brooklyn, NY, USA, in 1986.

He was an Academic Associate and an Assistant Professor with Polytechnic University from 1984 to 1986 and from 1986 to 1988, respectively. From 1988 to 1989, he was an Assistant Professor with the New Jersey Institute of Technology, Newark, NJ, USA. In 1989, he joined the RF System Division, European Space Research and Technology Centre (ESTEC), the European Space Agency, Noordwijk, The Netherlands, as a Senior Microwave Engineer, where he was in charge of the development of microwave filters and electromagnetic simulation tools. In 2001, he was appointed as the Head of the Technology Strategy Section, ESTEC, where he contributed to the development of management processes and tools for the formulation of a European strategy for the Space Technology Research and Development. In 2014, he retired from the European Space Agency, where he is currently holding the position of an Invited Senior Researcher at the Polytechnic University of Valencia, Valencia, Spain.

Dr. Guglielmi has been elevated to the grade of Fellow of the IEEE in January 2013 for contributions to multimode equivalent networks and microwave filter design. In 1981, he was awarded the Fulbright Scholarship in Rome, and an Halsey International Scholarship Programme (HISP) from the University of Bridgeport. 